\title{
Diastereoselective Synthesis of Cyclopropane Amino Acids Using Diazo Compounds Generated In Situ
}

Luke A. Adams, Varinder K. Aggarwal, * Roger V. Bonnert, ${ }^{\dagger}$ Bettina Bressel, Russell J. Cox, * Jon Shepherd, Javier de Vicente, Magnus Walter, ${ }^{*}$ William G. Whittingham, ${ }^{*}$ and Caroline L. Winn School of Chemistry, Bristol University, Cantock's Close, Bristol BS8 1TS, UK.

v.aggarwal@bristol.ac.uk

r.j.cox@bris.ac.uk

RECEIVED DATE (to be automatically inserted after your manuscript is accepted if required according to the journal that you are submitting your paper to)

$\dagger \quad$ AstraZeneca R\&D Charnwood, Medicinal Chemistry, Bakewell Road, Loughborough, Leics LE11 5RH, UK.

$\ddagger \quad$ Syngenta, Jealott's Hill International Research Centre, Bracknell, Berkshire RG42 6ET, UK.

* Corresponding Authors 


\section{Table of Contents}

1. General Methods 2 .

2. Characterisation of Tosylhydrazones 2 .

3. Characterisation of Tosylhydrazone Sodium Salts 4.

4. Synthesis and Characterisation of Dehydroamino Acids 5 .

5. Characterisation of Compounds in Table $1 \quad 8$.

6. Characterisation of Compounds in Table 29.

7. X-Ray Crystallographic Data 15 .

8. Spectra of new compounds 28 .

9. References 40.

\section{General Methods.}

All chemicals were purchased from common chemical suppliers and used as delivered unless otherwise stated. THF and toluene were pre-dried over sodium wire and then purified by passing through a solvent drying column prior to use. ${ }^{1}$ Dehydroamino acids $\mathbf{4}^{2} \mathbf{5}^{3}$ and $\mathbf{6}^{4}$ were prepared according to literature procedures (see also supplementary material). Commercially available dehydroamino acid 7 was either used as delivered or prepared using a newly developed large-scale procedure (see supplementary material).

\section{Characterisation of Tosylhydrazones.}

\section{Benzaldehyde tosylhydrazone. ${ }^{5}$}

Colourless needles (91\% yield); m.p. 127-128 ${ }^{\circ} \mathrm{C}(\mathrm{MeOH})\left(\right.$ lit.,$\left.{ }^{5} 128-129{ }^{\circ} \mathrm{C}\right) ;{ }^{1} \mathrm{H} \mathrm{NMR}(400 \mathrm{MHz}$, $\left.\mathrm{CDCl}_{3}\right) \delta 2.39\left(3 \mathrm{H}, \mathrm{s}, \mathrm{CH}_{3}\right), 7.29-7.36(5 \mathrm{H}, \mathrm{m}, \operatorname{Aryl} \mathrm{H}), 7.52-7.57(2 \mathrm{H}, \mathrm{m}, \operatorname{Aryl} \mathrm{H}), 7.78(1 \mathrm{H}, \mathrm{s}$, $\mathrm{C} H \mathrm{~N}), 7.89\left(2 \mathrm{H}, \mathrm{d}, J=8.3 \mathrm{~Hz}\right.$, Aryl H), $8.29\left(1 \mathrm{H}\right.$, br. s, NH); ${ }^{13} \mathrm{C}$ NMR $\left(100 \mathrm{MHz}, \mathrm{CDCl}_{3}\right) \delta 21.6$ (q), 127.3 (2d), 127.9 (2d), 128.6 (2d), 129.7 (2d), 130.4 (d), 133.1 (s), 135.2 (s), 144.3 (s), 147.9 (d).

p-Methoxybenzaldehyde tosylhydrazone. ${ }^{6}$

Colourless needles (83\% yield); m.p. 110-112 ${ }^{\circ} \mathrm{C}$ (lit., $\left.{ }^{6} 110-112{ }^{\circ} \mathrm{C}, \mathrm{MeOH}\right) ;{ }^{1} \mathrm{H} \mathrm{NMR}(400 \mathrm{MHz}$, $\left.\mathrm{CDCl}_{3}\right) \delta 2.39\left(3 \mathrm{H}, \mathrm{s}, \mathrm{ArCH}_{3}\right), 3.80\left(3 \mathrm{H}, \mathrm{s}, \mathrm{OCH}_{3}\right), 6.85(2 \mathrm{H}, \mathrm{d}, J=8.7 \mathrm{~Hz}, \operatorname{Aryl~H}), 7.29(2 \mathrm{H}, \mathrm{d}, J$ $=8.3 \mathrm{~Hz}, \operatorname{Aryl} \mathrm{H}), 7.50(2 \mathrm{H}, \mathrm{d}, J=8.7 \mathrm{~Hz}, \operatorname{Aryl~H}), 7.74(1 \mathrm{H}, \mathrm{s}, \mathrm{C} H \mathrm{~N}), 7.87(2 \mathrm{H}, \mathrm{d}, J=8.3 \mathrm{~Hz}$, Aryl H), 8.24 (1H, br. s, NH); ${ }^{13} \mathrm{C}$ NMR (100 MHz, $\left.\mathrm{CDCl}_{3}\right) \delta 21.6$ (q), 55.4 (q), 114.2 (2d), 126.1 (s), 128.0 (2d), 129.0 (2d), 129.7 (2d), 135.5 (s), 144.2 (s), 148.4 (d), 165.5 (s). 


\section{p-Methylbenzaldehyde tosylhydrazone. ${ }^{7}$}

Colourless needles (73\% yield); $\mathrm{R}_{f}=0.70$ (EtOAc/petroleum ether 1:4); m.p. 144-146 ${ }^{\circ} \mathrm{C}(\mathrm{MeOH})$ (lit. $\left.{ }^{7} 147-149{ }^{\circ} \mathrm{C}\right) ;{ }^{1} \mathrm{H}$ NMR $\left(270 \mathrm{MHz}, \mathrm{CDCl}_{3}\right) \delta 2.33\left(3 \mathrm{H}, \mathrm{s}, \mathrm{CH}_{3}\right), 2.38\left(3 \mathrm{H}, \mathrm{s}, \mathrm{CH}_{3}\right), 7.13(2 \mathrm{H}, \mathrm{d}$, $J=8.0 \mathrm{~Hz}, \operatorname{Aryl~H}), 7.28(2 \mathrm{H}, \mathrm{d}, J=8.0 \mathrm{~Hz}$, Aryl H), $7.46(2 \mathrm{H}, \mathrm{d}, J=8.0 \mathrm{~Hz}, \operatorname{Aryl~H}), 7.75(1 \mathrm{H}$, s, CH), $7.88\left(2 \mathrm{H}, \mathrm{d}, J=8.0 \mathrm{~Hz}\right.$, Aryl H), $8.24\left(1 \mathrm{H}\right.$, br. s, NH); ${ }^{13} \mathrm{C}$ NMR $\left(68 \mathrm{MHz}, \mathrm{CDCl}_{3}\right) \delta 21.5$ (q), 21.6 (q), 127.4 (2d), 127.9 (2d), 129.4 (2d), 129.7 (2d), 130.5 (s), 135.3 (s), 140.8 (s), 144.2 (s), $148.3(\mathrm{~d})$.

\section{p-Fluorobenzaldehyde tosylhydrazone. ${ }^{8}$}

Colourless needles (95\% yield); m.p. 133-134 ${ }^{\circ} \mathrm{C}(\mathrm{MeOH})\left(\mathrm{lit} .{ }^{8}, 134-136{ }^{\circ} \mathrm{C}\right) ;{ }^{1} \mathrm{H}$ NMR $(400 \mathrm{MHz}$, $\left.\mathrm{CDCl}_{3}\right) \delta 2.38\left(3 \mathrm{H}, \mathrm{s}, \mathrm{ArCH}_{3}\right), 7.04(2 \mathrm{H}, \mathrm{t}, J=8.4 \mathrm{~Hz}, \operatorname{Aryl~H}), 7.31(2 \mathrm{H}, \mathrm{d}, J=8.4 \mathrm{~Hz}$, Aryl H), $7.56\left(2 \mathrm{H}, \mathrm{dd}, J=8.4\right.$ and $\left.J_{\mathrm{H}-\mathrm{F}}=5.5 \mathrm{~Hz}, \operatorname{Aryl~H}\right), 7.77(1 \mathrm{H}, \mathrm{s}, \mathrm{NCH}), 7.84(2 \mathrm{H}, \mathrm{d}, J=8.4 \mathrm{~Hz}$, Aryl $\mathrm{H}), 8.56(1 \mathrm{H}$, br. s, $\mathrm{NH}) ;{ }^{13} \mathrm{C}$ NMR $\left(100 \mathrm{MHz}, \mathrm{CDCl}_{3}\right) \delta 21.2(\mathrm{q}), 115.5\left(2 \mathrm{~d}, J_{\mathrm{C}-\mathrm{F}}=22.3 \mathrm{~Hz}\right), 127.5$ (2d), $128.8\left(2 \mathrm{~d}, J_{\mathrm{C}-\mathrm{F}}=8.4 \mathrm{~Hz}\right), 129.4(2 \mathrm{~d}), 129.9(\mathrm{~s}), 135.9(\mathrm{~s}), 143.8(\mathrm{~s}), 146.1$ (d), $163.7\left(\mathrm{~s}, J_{\mathrm{C}-\mathrm{F}}\right.$ $=250.0 \mathrm{~Hz}) ; \mathrm{IR} \mathrm{v}_{\max } / \mathrm{cm}^{-1} 3437,1643,1602,1511,1167$.

\section{$\beta$-Phenyl-cinnamaldehyde tosyl hydrazone.}

Colourless needles (90\% yield); $\mathrm{R}_{f}=0.23$ (EtOAc/petroleum ether 1:4); m.p. 172-174 ${ }^{\circ} \mathrm{C}(\mathrm{MeOH})$ (lit. $\left.{ }^{9} 174-175{ }^{\circ} \mathrm{C}\right) ;{ }^{1} \mathrm{H}$ NMR $\left(400 \mathrm{MHz}, \mathrm{CDCl}_{3}\right) \delta 2.43\left(3 \mathrm{H}, \mathrm{s}, \mathrm{CH}_{3}\right), 6.79(1 \mathrm{H}, \mathrm{d}, J=9.8 \mathrm{~Hz}$, $\mathrm{NCHCH}), 7.12$ (2H, m, Aryl H), 7.21-7.40 (11H, m, Aryl H and NCHCH), $7.54(1 \mathrm{H}$, br. s, NH), $7.82\left(2 \mathrm{H}, \mathrm{m}\right.$, Aryl H); ${ }^{13} \mathrm{C}$ NMR (100 MHz, $\mathrm{CDCl}_{3}$ ) $\delta 21.7$ (q), 123.0 (d), 127.8 (2d), 128.0 (2d), 128.4 (d), 128.5 (2d), 128.7 (2d), 128.9 (d), 129.8 (2d), 130.3 (2d), 135.6 (s), 138.1 (s), 140.6 (s), $144.2(\mathrm{~s}), 148.2(\mathrm{~d}), 150.5(\mathrm{~s})$.

\section{3-[1-(tert-Butyl)-1,1-dimethylsilyl]oxybenzaldehyde. ${ }^{10}$}

Imidazole $(6.50 \mathrm{~g}, 95.5 \mathrm{mmol})$ and $\mathrm{TBSCl}(12 \mathrm{~g}, 79.6 \mathrm{mmol})$ were slowly added to a suspension of 3-hydroxybenzaldehyde $(9.72 \mathrm{~g}, 79.6 \mathrm{mmol})$ in anhydrous $\mathrm{CH}_{2} \mathrm{Cl}_{2}(190 \mathrm{~mL})$ at $0{ }^{\circ} \mathrm{C}$. After the addition, the reaction was stirred at $\mathrm{rt}$ for $3 \mathrm{~h}$. As phenol was still remaining (checked by TLC) another portion of $\mathrm{TBSCl}(1.80 \mathrm{~g}, 11.9 \mathrm{mmol})$ was added and the mixture was stirred for a further $16 \mathrm{~h}$. The resultant mixture was washed with a $2 \%$ aq. solution of $\mathrm{HCl}(2 \times 50 \mathrm{~mL})$, water $(2 \times 50$ $\mathrm{mL})$, brine $(2 \times 50 \mathrm{~mL})$ and then dried over $\mathrm{MgSO}_{4}$. After filtration, the solvent was removed under reduced pressure to afford 3-[1-(tert-butyl)-1,1-dimethylsilyl]oxybenzaldehyde (18.23 g, $97 \%$ ) as a colourless oil. ${ }^{11} \mathrm{R}_{f}=0.70$ (petroleum ether/EtOAc 3:1); ${ }^{1} \mathrm{H}$ NMR (400 MHz, $\left.\mathrm{CDCl}_{3}\right) \delta$ 
$0.23\left(6 \mathrm{H}, \mathrm{s}, \mathrm{Si}\left(\mathrm{CH}_{3}\right)_{2}\right), 1.00\left(9 \mathrm{H}, \mathrm{s}, \mathrm{SiC}\left(\mathrm{CH}_{3}\right)_{3}\right), 7.11(1 \mathrm{H}, \mathrm{dd}, J=7.8$ and $2.4 \mathrm{~Hz}$, Aryl H$), 7.33$ $(1 \mathrm{H}, \mathrm{d}, J=2.4 \mathrm{~Hz}$, Aryl H), $7.40(1 \mathrm{H}, \mathrm{dd}, J=7.8$ and $7.3 \mathrm{~Hz}, \operatorname{Aryl} \mathrm{H}), 7.48(1 \mathrm{H}, \mathrm{d}, J=7.3 \mathrm{~Hz}$, Aryl H), 9.96 (1H, s, CHO); ${ }^{13} \mathrm{C}$ NMR (100 MHz, $\left.\mathrm{CDCl}_{3}\right) \delta$-4.4 (2q), 18.3 (s), 25.7 (3q), 119.9 (d), 123.6 (d), 126.6 (d), 129.6 (d), 138.0 (s), 156.5 (s), 192.2 (d); GC-MS retention time 11.08 min, m/z (EI) $236\left(\mathrm{M}^{+}, 8\right), 179$ (100), 151 (40), 135 (7), 105 (4), 91 (7), 75 (8), 59 (5).

\section{3-(tert-Butyl-dimethyl-silyloxy)-benzaldehyde tosylhydrazone 23 . $^{12}$}

Colourless cubes (84\% yield); m.p. $120-122{ }^{\circ} \mathrm{C}(\mathrm{MeOH})\left(\mathrm{lit} .{ }^{12} 120-122{ }^{\circ} \mathrm{C}\right) ;{ }^{1} \mathrm{H}$ NMR $(400 \mathrm{MHz}$, $\left.\mathrm{CDCl}_{3}\right) \delta 0.19\left(6 \mathrm{H}, \mathrm{s}, \mathrm{Si}\left(\mathrm{CH}_{3}\right)_{3}\right), 0.98\left(9 \mathrm{H}, \mathrm{s}, \mathrm{SiC}\left(\mathrm{CH}_{3}\right)_{3}\right), 2.40\left(3 \mathrm{H}, \mathrm{s}, \mathrm{ArCH}{ }_{3}\right), 6.84(1 \mathrm{H}, \mathrm{dd}, J=$ 8.0 and $2.2 \mathrm{~Hz}$, Aryl H), $7.08(1 \mathrm{H}, \mathrm{d}, J=2.2 \mathrm{~Hz}$, Aryl H), $7.12(1 \mathrm{H}, \mathrm{d}, J=7.7 \mathrm{~Hz}$, Aryl H), 7.19 $(1 \mathrm{H}, \mathrm{dd}, J=8.0$ and $7.7 \mathrm{~Hz}$, Aryl H), $7.30(2 \mathrm{H}, \mathrm{d}, J=8.2 \mathrm{~Hz}, \operatorname{Aryl} \mathrm{H}), 7.71(1 \mathrm{H}, \mathrm{s}, \mathrm{NCH}), 7.88$ $\left(2 \mathrm{H}, \mathrm{d}, J=8.2 \mathrm{~Hz}\right.$, Aryl H), $8.21\left(1 \mathrm{H}\right.$, br. s, NH); ${ }^{13} \mathrm{C}$ NMR (100 MHz, $\left.\mathrm{CDCl}_{3}\right) \delta-4.4(2 \mathrm{q}), 18.3$ (s), 21.6 (q), 25.7 (3q), 118.3 (d), 121.0 (d), 122.5 (d), 128.1 (2d), 129.7 (d), 129.7 (2d), 134.7 (s), 135.4 (s), 144.3 (s), 147.8 (d), 156.0 (s); IR $v_{\max } / \mathrm{cm}^{-1}$ 3177, 1576, 1438, 1361, 1331; MS m/z (CI with $\left.\mathrm{CH}_{4}\right) 405$ ([MH] $\left.{ }^{+}, 20\right), 390$ (40), 107 (100). Anal Calc'd for $\mathrm{C}_{20} \mathrm{H}_{28} \mathrm{~N}_{2} \mathrm{O}_{3} \mathrm{SSi}$ : C, 59.37; H, 6.98; N, 6.92; found C, 59.25; H, 6.80; N, 7.06.

\section{2-Propenal tosylhydrazone $24 .^{12}$}

Yellow solid (68\% yield); eluent petroleum ether/EtOAc 35:65, $R_{\mathrm{f}}=0.29 ;$ m.p. $88-90{ }^{\circ} \mathrm{C}$ (petroleum ether/EtOAc) (lit. $\left.{ }^{12} 98-99{ }^{\circ} \mathrm{C}\right) ;{ }^{1} \mathrm{H} \mathrm{NMR}\left(400 \mathrm{MHz}, \mathrm{CDCl}_{3}\right) \delta 2.43\left(3 \mathrm{H}, \mathrm{s}, \mathrm{CH}_{3}\right), 5.52$ $(1 \mathrm{H}, \mathrm{d}, J=17.4 \mathrm{~Hz}, \mathrm{CHH}=\mathrm{CH}), 5.59(1 \mathrm{H}, \mathrm{d}, J=9.9 \mathrm{~Hz}, \mathrm{CH} H=\mathrm{CH}), 6.40(1 \mathrm{H}, \mathrm{dt}, J=17.4$ and 9.9 $\mathrm{Hz}, \mathrm{CHH}=\mathrm{CH}), 7.28-7.36(2 \mathrm{H}, \mathrm{m}$, Aryl H$), 7.41(1 \mathrm{H}, \mathrm{d}, J=9.9 \mathrm{~Hz}, \mathrm{CHN}), 7.78-7.86(2 \mathrm{H}, \mathrm{m}$, Aryl $\mathrm{H}), 8.06(1 \mathrm{H}$, br. s, $\mathrm{NH}) ;{ }^{13} \mathrm{C} \mathrm{NMR}\left(100 \mathrm{MHz}, \mathrm{CDCl}_{3}\right) \delta 21.7(\mathrm{q}), 125.2(\mathrm{t}), 127.9(2 \mathrm{~d}), 129.8(2 \mathrm{~d})$, 133.2 (d), 135.3 (s), 144.3 (s), 149.8 (d); IR $v_{\max } / \mathrm{cm}^{-1}$ 1597, 1344, 1158, 936, 809; MS m/z (ES) $225\left([\mathrm{MH}]^{+}, 100\right)$. Anal. Calc'd for $\mathrm{C}_{10} \mathrm{H}_{12} \mathrm{~N}_{2} \mathrm{O}_{2} \mathrm{~S}: \mathrm{C}, 53.5 ; \mathrm{H}, 5.4 ; \mathrm{N}, 12.5$. Found: C, 53.6; H, 5.5; $\mathrm{N}, 12.4$.

\section{Characterisation of Tosylhydrazone Sodium Salts.}

\section{Benzaldehyde tosylhydrazone sodium salt. ${ }^{13}$}

Colourless powder; ${ }^{1} \mathrm{H}$ NMR (400 MHz, $\left.\mathrm{D}_{2} \mathrm{O}\right) \delta 2.17\left(3 \mathrm{H}, \mathrm{s}, \mathrm{CH}_{3}\right), 7.15-7.23(5 \mathrm{H}, \mathrm{m}$, Aryl H), 7.35-7.40 (2H, m, Aryl H), $7.60\left(2 \mathrm{H}, \mathrm{d}, J=8.4 \mathrm{~Hz}\right.$, Aryl H), $7.83(1 \mathrm{H}, \mathrm{s}, \mathrm{CHN}) ;{ }^{13} \mathrm{C}$ NMR $(100$ $\left.\mathrm{MHz}, \mathrm{D}_{2} \mathrm{O}\right) \delta 20.7(\mathrm{q}), 126.6$ (2d), 126.7 (2d), 128.8 (2d), 129.5 (2d), 129.6 (d), 135.8 (s), 139.2 (s), 142.8 (s), 145.9 (d); MS m/z (FAB) $297\left([\mathrm{MH}]^{+}, 84\right)$. 


\section{p-Methylbenzaldehyde tosylhydrazone sodium salt. ${ }^{\top}$}

Pink solid; ${ }^{1} \mathrm{H}$ NMR (270 MHz, $\left.\mathrm{D}_{2} \mathrm{O}\right) \delta 2.13\left(3 \mathrm{H}, \mathrm{s}, \mathrm{CH}_{3}\right), 2.26\left(3 \mathrm{H}, \mathrm{s}, \operatorname{ArCH}_{2}\right), 7.11(2 \mathrm{H}, \mathrm{d}, J=8.0$ $\mathrm{Hz}$, Aryl H), $7.26(2 \mathrm{H}, \mathrm{d}, J=8.0 \mathrm{~Hz}$, Aryl H), $7.37(2 \mathrm{H}, \mathrm{d}, J=8.0 \mathrm{~Hz}, \operatorname{Aryl~H}), 7.70(2 \mathrm{H}, \mathrm{d}, J=$ $8.0 \mathrm{~Hz}$, Aryl H), 7.89 (1H, s, NCH); ${ }^{13} \mathrm{C}$ NMR (75 MHz, D $\left.\mathrm{O}\right) \delta 20.3$ (q), 20.5 (q), 126.4 (2d), 126.5 (2d), 129.2 (2d), 129.3 (2d), 132.7 (s), 139.2 (d), 142.5 (s), 146.0 (s); MS m/z (FAB) 311 ([MH $\left.]^{+}, 41\right), 308$ (25), 307 (100); HRMS (FAB, $\left.[\mathrm{MH}]^{+}\right)$: found $311.0834 \mathrm{C}_{15} \mathrm{H}_{16} \mathrm{~N}_{2} \mathrm{O}_{2} \mathrm{SNa}$ requires 311.0830 .

\section{p-Methoxybenzaldehyde tosylhydrazone sodium salt. ${ }^{7}$}

Colourless solid; ' $\mathrm{H}$ NMR (270 MHz, D 20$) \delta 2.20\left(3 \mathrm{H}, \mathrm{s}, \mathrm{ArCH}_{3}\right), 3.65\left(3 \mathrm{H}, \mathrm{s}, \mathrm{OCH}_{3}\right), 6.78(2 \mathrm{H}$, d, $J=8.0$, Aryl H), $7.19(2 \mathrm{H}, \mathrm{d}, J=8.0 \mathrm{~Hz}, \operatorname{Aryl~H}), 7.36(2 \mathrm{H}, \mathrm{d}, J=8.0 \mathrm{~Hz}$, Aryl H), $7.68(2 \mathrm{H}, \mathrm{d}$, $J=8.0 \mathrm{~Hz}, \operatorname{Aryl} \mathrm{H}), 7.86(1 \mathrm{H}, \mathrm{s}, \mathrm{NCH}) ;{ }^{13} \mathrm{C} \mathrm{NMR}\left(75 \mathrm{MHz}, \mathrm{D}_{2} \mathrm{O}\right) \delta 20.4$ (q), 55.2 (q), 113.9 (2d), 126.5 (2d), 127.8 (s), 128.7 (2d), 129.3 (2d), 139.3 (s), 142.4 (s), 145.6(d), 159.1 (s); MS m/z (FAB) $327\left([\mathrm{MH}]^{+}, 100\right), 298(40)$; $\mathrm{HRMS}\left(\mathrm{FAB},[\mathrm{MH}]^{+}\right)$: found $327.0779 \mathrm{C}_{15} \mathrm{H}_{16} \mathrm{~N}_{2} \mathrm{O}_{3} \mathrm{SNa}$ requires 327.0799 .

\section{p-Fluorobenzaldehyde tosylhydrazone sodium salt. ${ }^{12}$}

Pink solid; ${ }^{1} \mathrm{H}$ NMR (400 MHz, $d_{6}$-DMSO) $\delta 2.27\left(3 \mathrm{H}, \mathrm{s}, \mathrm{ArCH}_{3}\right), 7.05\left(2 \mathrm{H}, \mathrm{t}, J=8.8 \mathrm{~Hz}\right.$ and $J_{H-F}$ $=8.8 \mathrm{~Hz}$, Aryl H), $7.16\left(2 \mathrm{H}, \mathrm{d}, J=8.0 \mathrm{~Hz}\right.$, Aryl H), $7.41\left(2 \mathrm{H}, \mathrm{dd}, J=8.8 \mathrm{~Hz}\right.$ and $J_{H-F}=6.6 \mathrm{~Hz}$,

Aryl H), $7.65\left(2 \mathrm{H}, \mathrm{d}, J=8.0 \mathrm{~Hz}\right.$, Aryl H), $7.66(1 \mathrm{H}, \mathrm{s}, \mathrm{NCH}) ;{ }^{13} \mathrm{C}$ NMR (100 MHz, $d_{6}$-DMSO) $\delta$ $21.4(\mathrm{q}), 115.5\left(2 \mathrm{~d}, J_{C-F}=21.4 \mathrm{~Hz}\right), 126.9\left(2 \mathrm{~d}, J_{C-F}=7.7 \mathrm{~Hz}\right), 127.1(2 \mathrm{~d}), 128.8(2 \mathrm{~d}), 135.4(\mathrm{~s})$, 136.6 (d), 139.2 (s), 144.4 (s), 161.4 (s, $J_{C-F}=241.6 \mathrm{~Hz}$ ); IR $v_{\max } / \mathrm{cm}^{-1} 1602,1508,1225,1126$, 1084, 1033, 952, 776; MS m/z (CI with $\left.\mathrm{CH}_{4}\right) 315\left([\mathrm{MH}]^{+}, 12\right)$.

\section{$\beta$-Phenyl-cinnamaldehyde tosylhydrazone sodium salt. ${ }^{12}$}

Pale orange solid; ${ }^{1} \mathrm{H}$ NMR (400 MHz, CD $\left.\mathrm{OD}\right) \delta 2.30\left(3 \mathrm{H}, \mathrm{s}, \mathrm{CH}_{3}\right), 6.80(1 \mathrm{H}, \mathrm{d}, J=10.0 \mathrm{~Hz}$, CHCHN), 7.05-7.34 (10H, m, Aryl H), 7.43-7.51 (2H, m, Aryl H), $7.54(1 \mathrm{H}, \mathrm{d}, J=10.0 \mathrm{~Hz}$, $\mathrm{CH}=\mathrm{CHN}$ ) and 7.63-7.74 (2H, m, Aryl H); ${ }^{13} \mathrm{C}$ NMR (75 MHz, CD 3 OD) $\delta 20.0(\mathrm{q}), 123.9(\mathrm{~d})$, 126.8 (2d), 126.9 (2d), 127.1 (d), 127.2 (d), 127.9 (2d), 128.0 (2d), 128.5 (2d), 130.2 (2d), 139.6 (s), 140.7 (s), 141.8 (s), 142.0 (s), 143.4 (s), 144.1 (d); IR $v_{\max } / \mathrm{cm}^{-1} 1062,811,764,695$; MS m/z (CI with $\left.\mathrm{CH}_{4}\right) 384$ ([MH-Me] $\left.]^{+}, 1\right), 323$ (1), 307 (3), 221 (41), 206 (17), 193 (100), 91 (22); HRMS $\left(\mathrm{CI},[\mathrm{MH}-\mathrm{Ph}+\mathrm{H}]^{+}\right)$Found: 323.0834. Calc. for $\mathrm{C}_{16} \mathrm{H}_{16} \mathrm{~N}_{2} \mathrm{O}_{2} \mathrm{SNa} 323.0830$.

$$
-5 \text { - }
$$




\section{Synthesis and Characterisation of Dehydroaminoacids.}

\section{Methyl 2-[(tert-butoxycarbonyl)amino]acrylate $4 .^{2}$}

EDCI (11.40 g, $59.5 \mathrm{mmol})$ was added under nitrogen to a mixture of $N$-(tert-butoxycarbonyl)$D L$-serine methyl ester $(12.42 \mathrm{~g}, 56.7 \mathrm{mmol})$ and $\mathrm{CuCl}(5.89 \mathrm{~g}, 59.48 \mathrm{mmol})$ in anhydrous $\mathrm{CH}_{2} \mathrm{Cl}_{2}$ $(100 \mathrm{~mL})$ at $0^{\circ} \mathrm{C}$. After stirring at $\mathrm{rt}$ in a dark fume cupboard for $60 \mathrm{~h}$, the solvent was removed under reduced pressure and the oily residue was dissolved in EtOAc $(2 \mathrm{~L})$, washed with water $(2 \times$ $400 \mathrm{~mL})$ and brine $(300 \mathrm{~mL})$ and dried over $\mathrm{Na}_{2} \mathrm{SO}_{4}$. After filtration, the solvent was evaporated under reduced pressure to give a residue. The crude product was purified by flash chromatography (eluent petroleum ether/EtOAc 10:1) to give methyl 2-[(tertbutoxycarbonyl)amino]acrylate $4(7.81 \mathrm{~g}, 69 \%)$ as a colourless oil. The product was stored at $\mathrm{rt}$ in a dark cupboard and polymerised after a few days; $\mathrm{R}_{f}=0.80$ (petroleum ether/EtOAc 10:1); ${ }^{1} \mathrm{H}$ $\operatorname{NMR}\left(400 \mathrm{MHz}, \mathrm{CDCl}_{3}\right) \delta 1.49\left(9 \mathrm{H}, \mathrm{s} \mathrm{C}\left(\mathrm{CH}_{3}\right)_{3}\right), 3.83\left(3 \mathrm{H}, \mathrm{s}, \mathrm{COOCH}_{3}\right), 5.73(1 \mathrm{H}, \mathrm{s}, \mathrm{CHH}), 6.16$ $(1 \mathrm{H}, \mathrm{s}, \mathrm{CH} H), 7.02(1 \mathrm{H}$, br. s, $\mathrm{NH}) ;{ }^{13} \mathrm{C} \mathrm{NMR}\left(100 \mathrm{MHz}, \mathrm{CDCl}_{3}\right) \delta 28.3$ (3q), 52.9 (q), 80.8 (s), $105.2(\mathrm{t}), 131.4(\mathrm{~s}), 152.6(\mathrm{~s}), 164.5(\mathrm{~s})$.

\section{4-Nitrobenzyl 2-[(tert-butoxycarbonyl)amino]-3-hydroxypropanoate. ${ }^{14}$}

$N$-(tert-Butoxycarbonyl)- $D L$-serine $(26.6 \mathrm{~g}, 130 \mathrm{mmol})$ was dissolved in $\mathrm{MeOH}(500 \mathrm{~mL})$ and water $(50 \mathrm{~mL})$. The $\mathrm{pH}$ of this weakly acidic solution was ajusted to $\mathrm{pH}=7$ with a $20 \%$ aq. solution of cesium carbonate. The solvent was removed under reduced pressure give the $N$-(tertbutoxycarbonyl)- $D L$-serine cesium salt as a colourless viscous oil. 4-Nitrobenzyl bromide (39.2 g, $182 \mathrm{mmol}$ ) was added to a solution of the cesium salt in DMF $(300 \mathrm{~mL})$ and the resultant mixture stirred at $\mathrm{rt}$ for $24 \mathrm{~h}$. Water $(200 \mathrm{~mL})$ and $\mathrm{CH}_{2} \mathrm{Cl}_{2}(500 \mathrm{~mL})$ were added to the reaction mixture. The organic layer was separated and washed with water $(4 \times 200 \mathrm{~mL})$, dried over $\mathrm{Na}_{2} \mathrm{SO}_{4}$, filtered and evaporated under reduced pressure to give a yellow oily residue, which solidified on standing. The crude material was recrystallised from EtOAc/hexane to give the ester 4-nitrobenzyl 2-[(tertbutoxycarbonyl)amino]-3-hydroxypropanoate $(27.9 \mathrm{~g}, 63 \%)$ as colourless crystals. ${ }^{1} \mathrm{H}$ NMR (300 $\left.\mathrm{MHz}, \mathrm{CDCl}_{3}\right) \delta 1.45\left(9 \mathrm{H}, \mathrm{s}, \mathrm{C}\left(\mathrm{CH}_{3}\right)_{3}\right), 2.44-2.56(1 \mathrm{H}, \mathrm{m}, \mathrm{OH}), 3.94(1 \mathrm{H}, \mathrm{ddd}, J=11.2,5.5$ and 3.5 $\mathrm{Hz}, \mathrm{CHHOH}), 4.05(1 \mathrm{H}, \mathrm{ddd}, J=11.0,5.5$ and $3.7 \mathrm{~Hz}, \mathrm{CH} H \mathrm{OH}), 4.40-4.52(1 \mathrm{H}, \mathrm{m}, \mathrm{CHNH}), 5.28$ $(1 \mathrm{H}, \mathrm{d}, J=13.5 \mathrm{~Hz}, \mathrm{CHHAr}), 5.35(1 \mathrm{H}, \mathrm{d}, J=13.5 \mathrm{~Hz}, \mathrm{CH} H \mathrm{Ar}), 5.51(1 \mathrm{H}$, br. d, $J=7.7 \mathrm{~Hz}, \mathrm{NH})$, 7.49-7.57 (2H, m, Aryl H), 8.18-8.26 (2H, m, Aryl H); ${ }^{13} \mathrm{C}$ NMR (75 MHz, $\left.\mathrm{CDCl}_{3}\right) \delta 28.2(3 \mathrm{q})$, $55.7(\mathrm{~d}), 63.3(\mathrm{t}), 65.7(\mathrm{t}), 80.5(\mathrm{~s}), 123.8(2 \mathrm{~d}), 128.2(2 \mathrm{~d}), 142.5(\mathrm{~s}), 147.7(\mathrm{~s}), 155.7(\mathrm{~s}), 170.6(\mathrm{~s})$; 
IR $v_{\max } / \mathrm{cm}^{-1} 3414,3345,1748,1665,1607,1523,1350,1292,856 ; \mathrm{MS} \mathrm{m} / z(\mathrm{CI}) 341\left([\mathrm{MH}]^{+}, 15\right)$, 285 (66), 267 (18), 241 (100), 160 (16), 136 (24), 122 (14) and 57 (54).

\section{4-Nitrobenzyl 2-[(tert-butoxycarbonyl)amino]acrylate $5{ }^{14}$}

EDCI $(29.2 \mathrm{~g}, 152 \mathrm{mmol})$ was added under nitrogen to a mixture of 4-nitrobenzyl 2-[(tertbutoxycarbonyl)amino]-3-hydroxypropanoate $(5.9 \mathrm{~g}, 76.1 \mathrm{mmol})$ and $\mathrm{CuCl}(753 \mathrm{mg}, 7.61 \mathrm{mmol})$ in anhydrous $\mathrm{CHCl}_{3}(700 \mathrm{~mL})$ at $\mathrm{rt}$. The mixture rapidly became a dark brown colour and was stirred for $17 \mathrm{~h}$ at $\mathrm{rt}$. The reaction mixture was filtered through a celite ${ }^{\circledR}$ pad and washed with brine $(700 \mathrm{~mL})$, before the layers were separated and the aq. extracted with $\mathrm{CHCl}_{3}(3 \times 500 \mathrm{~mL})$. The combined organic layers were dried over $\mathrm{MgSO}_{4}$, filtered and evaporated under reduced pressure to give 4-nitrobenzyl 2-[(tert-butoxycarbonyl)amino]acrylate $(24.2 \mathrm{~g}, 99 \%)$ as an off white solid; $\mathrm{R}_{f}=0.70$ (petroleum ether/EtOAc 10:1); ${ }^{1} \mathrm{H} \mathrm{NMR}\left(270 \mathrm{MHz}, \mathrm{CDCl}_{3}\right) \delta 1.49(9 \mathrm{H}$, s, $\left.\mathrm{C}\left(\mathrm{CH}_{3}\right)_{3}\right), 5.36\left(2 \mathrm{H}, \mathrm{s}, \mathrm{COOCH}_{2}\right), 5.83(1 \mathrm{H}, \mathrm{s}, \mathrm{CHH}=\mathrm{CH}), 6.25(1 \mathrm{H}, \mathrm{s}, \mathrm{CH} H=\mathrm{CH}), 6.99(1 \mathrm{H}$, br. s, NH), 7.50-7.58 (2H, m, Aryl H), 8.21-8.29 (2H, m, Aryl H); IR $v_{\max } / \mathrm{cm}^{-1} 3426,1735,1712,1640$, 1606, 1515, 886, 840; MS m/z (CI with $\left.\mathrm{CH}_{4}\right) 323\left([\mathrm{MH}]^{+}, 2\right), 57$ (100).

\section{Methyl 2-[di(tert-butoxycarbonyl)amino]acrylate $6 .^{15}$}

To a solution of $N$-(tert-butoxycarbonyl)- $D L$-serine methyl ester (9.69 g, $44.2 \mathrm{mmol})$ in anhydrous MeCN (175 mL) was added DMAP (540 mg, $4.42 \mathrm{mmol}$ ) followed by di-tert-butyl dicarbonate (21.2 g, $97.2 \mathrm{mmol})$. The colourless solution was heated at gentle reflux under nitrogen for $8 \mathrm{~h}$. After removal of the solvent, diethyl ether $(200 \mathrm{~mL})$ and water $(100 \mathrm{~mL})$ were added to the resultant residue. The aq. layer was acidified to $\mathrm{pH}=1$ with a saturated solution of aq. $\mathrm{KHSO}_{4}$ and the layers were separated. The organic layer was washed with water $(100 \mathrm{~mL})$, a saturated solution of aq. $\mathrm{KHSO}_{4}(3 \times 100 \mathrm{~mL})$, a saturated solution of aq. $\mathrm{NaHCO}_{3}(3 \times 100 \mathrm{~mL})$ and brine $(3 \times 100$ $\mathrm{mL}$ ). The organic layer was separated and dried over $\mathrm{Na}_{2} \mathrm{SO}_{4}$. After filtration, the solvent was evaporated under reduced pressure to give methyl 2-[di(tert-butoxycarbonyl)amino]acrylate (12.3 $\mathrm{g}, 93 \%$ ) as a pale brown solid; $\mathrm{R}_{f}=0.40$ (petroleum ether/EtOAc 3:1); m.p. 54-55 ${ }^{\circ} \mathrm{C}$ (lit., ${ }^{15}$ 52$\left.52.5{ }^{\circ} \mathrm{C}\right) ;{ }^{1} \mathrm{H} \mathrm{NMR}\left(270 \mathrm{MHz}, \mathrm{CDCl}_{3}\right) \delta 1.47\left(18 \mathrm{H}, \mathrm{s}, 2 \mathrm{C}\left(\mathrm{CH}_{3}\right)_{3}\right), 3.80\left(3 \mathrm{H}, \mathrm{s}, \mathrm{COOCH}_{3}\right), 5.65(1 \mathrm{H}$, s, $\mathrm{CHH}=\mathrm{CH}), 6.35(1 \mathrm{H}, \mathrm{s}, \mathrm{CH} H=\mathrm{CH}) . m / z(\mathrm{ES}) 324$ ([MNa] $\left.]^{+}, 98 \%\right), 302$ (86), 287 (20), 272 (15), $246(100)$.

\section{Methyl 2-(acetylamino)acrylate $7 .^{16}$}

A mixture of serine methyl ester hydrochloride $(12.5 \mathrm{~g}, 80.3 \mathrm{mmol})$, anhydrous sodium acetate (39.54 g, $482 \mathrm{mmol})$ and acetic anhydride $(120 \mathrm{~mL})$ was heated at reflux for $2 \mathrm{~h}$. The volatiles were evaporated under reduced pressure and the residue was dissolved in $\mathrm{Et}_{2} \mathrm{O}(200 \mathrm{~mL})$. A 
saturated aq. solution of $\mathrm{NaHCO}_{3}(200 \mathrm{~mL})$ was added and the biphasic mixture was stirred for 1 h. The organic layer was separated and washed with a saturated aq. solution of $\mathrm{NaHCO}_{3}(40 \mathrm{~mL})$. The organic layer was separated and dried over $\mathrm{Na}_{2} \mathrm{SO}_{4}$. After filtration, the solvent was removed under reduced pressure to give a 83:17 mixture of methyl 2-(diacetylamino)acrylate $(9.05 \mathrm{~g}, 61 \%)$ and methyl 2-(acetylamino)acrylate $(1.43 \mathrm{~g}, 12 \%)$ as a yellow oil. Methyl 2(diacetylamino)acrylate; ${ }^{1} \mathrm{H}$ NMR $\left(400 \mathrm{MHz} \mathrm{CDCl}_{3}\right) \delta 2.36\left(6 \mathrm{H}, \mathrm{s}, 2 \times \mathrm{CH}_{3} \mathrm{CON}\right), 3.83(3 \mathrm{H}, \mathrm{s}$, $\left.\mathrm{COOCH}_{3}\right), 5.81(1 \mathrm{H}, \mathrm{s} \mathrm{CHH}), 6.60(1 \mathrm{H}, \mathrm{s}, \mathrm{CH} H) ;{ }^{13} \mathrm{C} \mathrm{NMR}\left(100 \mathrm{MHz}, \mathrm{CDCl}_{3}\right) \delta 26.0(2 \mathrm{q}), 53.0$ (q), 128.1 (t), $137.3(\mathrm{~s}), 163.4(\mathrm{~s}), 172.2$ (2s); GC-MS retention time $7.21 \mathrm{~min} ., \mathrm{m} / \mathrm{z}$ (EI) $185\left(\mathrm{M}^{+}\right.$, 1), 143 (56), 111 (32), 101 (100), 71 (20).

The crude reaction mixture was dissolved in $\mathrm{MeOH}(150 \mathrm{~mL})$ and treated with triethylamine $(1.57 \mathrm{~mL}, 11.3 \mathrm{mmol})$ and hydroquinone $(0.19 \mathrm{~g}, 1.7 \mathrm{mmol})$. The reaction mixture was heated at reflux until all methyl 2-(diacetylamino)acrylate was consumed (followed by TLC and GC-MS). The solvent was removed under reduced pressure and the resultant solid was triturated in cyclohexane. The cyclohexane-acetic acid azeotropic mixture was evaporated under reduced pressure and the remaining solid was recrystallysed in diethyl ether $(165 \mathrm{~mL})$ to give methyl 2-(acetylamino)acrylate (7.94 g, 70\%) as colourless needles. ${ }^{1} \mathrm{H}$ NMR $\left(400 \mathrm{MHz}, \mathrm{CDCl}_{3}\right) \delta$ $2.14\left(3 \mathrm{H}, \mathrm{s}, \mathrm{CH}_{3} \mathrm{CONH}\right), 3.85\left(3 \mathrm{H}, \mathrm{s}, \mathrm{COOCH}_{3}\right), 5.89(1 \mathrm{H}, \mathrm{s} \mathrm{CHH}), 6.61(1 \mathrm{H}, \mathrm{s}, \mathrm{CH} H), 7.74(1 \mathrm{H}$, br. s, $\mathrm{NH}$ ); ${ }^{13} \mathrm{C}$ NMR (100 MHz, $\mathrm{CDCl}_{3}$ ) $\delta 24.7$ (q), 53.0 (q), 108.8 (t), 130.9 (s), 164.7 (s), 168.9 (s); GC-MS retention time 7.05 min., m/z (EI) $143\left(\mathrm{M}^{+}, 55\right), 111$ (31), 101 (100), 71 (20).

\section{Characterisation of Compounds from Table 1 - yields and ratios given in Table 1.}

\section{Methyl $\left(1 S^{*}, \quad 2 R^{*}\right)-\quad$ and $\left(1 R^{*}, \quad 2 R^{*}\right)-1-[($ tert-butoxycarbonyl)amino]-2-phenyl-1-} cyclopropanecarboxylate 8 (Table 1, entries 1-5). ${ }^{17}$

$\boldsymbol{E - 8}$; colourless solid; eluent petroleum ether/EtOAc 3:1, $\mathrm{R}_{f}=0.52$; m.p. $87-89{ }^{\circ} \mathrm{C}$ (petroleum ether/EtOAc) (lit., $\left.{ }^{17} 85-86{ }^{\circ} \mathrm{C}\right) ;{ }^{1} \mathrm{H}$ NMR $\left(400 \mathrm{MHz}, \mathrm{CDCl}_{3}\right) \delta 1.49\left(9 \mathrm{H}, \mathrm{s}, \mathrm{C}\left(\mathrm{CH}_{3}\right)_{3}\right), 1.61(1 \mathrm{H}, \mathrm{m}$, $\mathrm{CH} H), 2.18(1 \mathrm{H}, \mathrm{dd}, J=8.4$ and $5.5 \mathrm{~Hz}, \mathrm{CH} H), 2.84(1 \mathrm{H}, \mathrm{dd}, J=10.1$ and $8.4 \mathrm{~Hz}, \mathrm{CHPh}), 3.35$ $\left(3 \mathrm{H}, \mathrm{s}, \mathrm{CH}_{3} \mathrm{O}\right), 5.33(1 \mathrm{H}$, br. s, $\mathrm{NH}), 7.07-7.51(5 \mathrm{H}, \mathrm{m}$, Aryl H$) ;{ }^{13} \mathrm{C}-\mathrm{NMR}\left(100 \mathrm{MHz}, \mathrm{CDCl}_{3}\right) \delta$ $20.3(\mathrm{t}), 28.4$ (3q), 35.2 (d), 40.6 (s), 51.9 (q), 76.8 (s), 127.0 (d), 128.0 (2d), 129.3 (2d), 135.4 (s), 156.0 (s), 170.7 (s); IR $v_{\max } / \mathrm{cm}^{-1} 3343,2987,1735,1682,1494,1283,1150,700$; GC-MS retention time 13.84 min., $m / z$ (EI) 235 ([M- $t$-Bu $\left.]^{+}, 5\right), 159$ (35), 131 (36), 104 (13), 77 (8), 57 (100).

Z-8; colourless solid; eluent petroleum ether/EtOAc 3:1, $\mathrm{R}_{f}=0.48$; m.p. 107-109 ${ }^{\circ} \mathrm{C}$ (petroleum ether/EtOAc); ${ }^{1} \mathrm{H}$ NMR $\left(300 \mathrm{MHz}, 124{ }^{\circ} \mathrm{C}, d_{6}\right.$-DMSO) $\delta 1.16\left(9 \mathrm{H}, \mathrm{s}, \mathrm{C}\left(\mathrm{CH}_{3}\right)_{3}\right), 1.63(1 \mathrm{H}, \mathrm{dd}, J=$ 
8.3 and $5.7 \mathrm{~Hz}, \mathrm{CH} H), 1.69(1 \mathrm{H}, \mathrm{dd}, J=9.7$ and $5.7 \mathrm{~Hz}, \mathrm{CH} H), 2.92(1 \mathrm{H}, \mathrm{dd}, J=9.7$ and $8.3 \mathrm{~Hz}$, $\mathrm{CHPh}), 3.65\left(3 \mathrm{H}, \mathrm{s}, \mathrm{CH}_{3} \mathrm{O}\right), 6.47\left(1 \mathrm{H}\right.$, br. s, NH), 7.14-7.25 (5H, m, Aryl H); ${ }^{13} \mathrm{C}$ NMR $(75 \mathrm{MHz}$, $124^{\circ} \mathrm{C}, d_{6}$-DMSO) $\delta 21.0(\mathrm{t}), 28.4(3 \mathrm{q}), 32.2(\mathrm{~d}), 39.9(\mathrm{~s}), 52.4(\mathrm{q}), 78.7(\mathrm{~s}), 126.8(\mathrm{~d}), 128.0(2 \mathrm{~d})$, 129.2 (2d), 136.1 (s), 155.6 (s), 172.9 (s); IR $v_{\max } / \mathrm{cm}^{-1}$ 3368, 2986, 1722, 1691, 1505, 1287, 1243, 1158, 769, 697; GC-MS retention time $13.91 \mathrm{~min} ., \mathrm{m} / z$ (EI) 235 ([M- $t$-Bu] $\left.]^{+}, 7\right), 159$ (45), 131 (47), 104 (15), 77 (9), 57 (100).

$\left(1 R^{*}, 2 S^{*}\right)-$ and $\left(1 R^{*}, 2 R^{*}\right)-4-N i t r o b e n z y l \quad 1-[($ tert-butoxycarbonyl)amino]-2-phenyl-1cyclopropanecarboxylate 9 (Table 1, entries 6-7).

See experimental section in paper.

Methyl $\left(1 R^{*}, 2 S^{*}\right)$ - and $\left(1 R^{*}, 2 R^{*}\right)$-1-(acetylamino)-2-phenyl-1-cyclopropanecarboxylate 10 (Table 1, entries 10-12). ${ }^{18}$

See experimental section in paper.

6. Characterisation of Compounds in Table 2 - yields and ratios given in Table 2.

Table 2, entry 1, compound 9 - see Table 1, entries 6-7.

Table 2, entry 2, compound 10 - see Table 1, entries 10-12.

$\left(1 R^{*}, \quad 2 S^{*}\right)-\quad$ and $\quad\left(1 R^{*}, \quad 2 R^{*}\right)-4-$ Nitrobenzyl 1-[(tert-butoxycarbonyl)amino]-2-(4methoxyphenyl)-1-cyclopropanecarboxylate 12 (Table 2, entry 3).

$\boldsymbol{E - 1 2}$; colourless solid; eluent petroleum ether/EtOAc 3:1, $\mathrm{R}_{f}=0.25$; m.p. $129-131{ }^{\circ} \mathrm{C}$ (EtOAc/petroleum ether); ${ }^{1} \mathrm{H}$ NMR $\left(400 \mathrm{MHz}, \mathrm{CDCl}_{3}\right) \delta 1.47\left(9 \mathrm{H}, \mathrm{s}, \mathrm{C}\left(\mathrm{CH}_{3}\right)_{3}\right), 1.63(1 \mathrm{H}, \mathrm{dd}, J=$ 9.2 and $\left.5.5 \mathrm{~Hz}, \mathrm{CHHCCO}_{2}\right), 2.17\left(1 \mathrm{H}, \mathrm{dd}, J=8.4\right.$ and $\left.5.5 \mathrm{~Hz}, \mathrm{C} H \mathrm{HCCO}_{2}\right), 2.85(1 \mathrm{H}, \mathrm{dd}, J=9.2$ and $8.4 \mathrm{~Hz}, \mathrm{ArCH}), 3.75\left(3 \mathrm{H}, \mathrm{s}, \mathrm{ArOCH}_{3}\right), 4.95\left(2 \mathrm{H}, \mathrm{s}, \mathrm{COOCH}_{2}\right), 5.37(1 \mathrm{H}$, br. s, NH), $6.73(2 \mathrm{H}$, $\mathrm{d}, J=8.8 \mathrm{~Hz}$, Aryl H), $7.11(2 \mathrm{H}, \mathrm{d}, J=8.8 \mathrm{~Hz}$, Aryl H), 7.22 (2H, br. d, $J=8.4 \mathrm{~Hz}$, Aryl H), 8.07 $\left(2 \mathrm{H}, \mathrm{d}, J=8.4 \mathrm{~Hz}\right.$, Aryl H); ${ }^{13} \mathrm{C} \mathrm{NMR}\left(100 \mathrm{MHz}, \mathrm{CDCl}_{3}\right) \delta 21.1(\mathrm{t}), 28.4(3 \mathrm{q}), 35.1(\mathrm{~d}), 41.4$ (s), $55.2(\mathrm{q}), 65.2(\mathrm{t}), 80.2(\mathrm{~s}), 113.5(2 \mathrm{~d}), 123.4(2 \mathrm{~d}), 127.2$ (s), 128.3 (2d), 130.4 (2d), 142.9 (s), 147.5 (s), 156.0 (s), 158.8 (s), 170.0 (s); IR v max $/ \mathrm{cm}^{-1}$ 3242, 3126, 2977, 1701, 1610, 1515, 1454, 1390, 1366, 1344, 1249, 1150, 1058, 1037, 925, 852, 805, 701; MS m/z (EI) $443\left([\mathrm{MH}]^{+}, 30\right), 343$ (19), 250 (70), 206 (100), 189 (90). Anal. Calc'd for $\mathrm{C}_{23} \mathrm{H}_{26} \mathrm{~N}_{2} \mathrm{O}_{7}: \mathrm{C}, 62.43 ; \mathrm{H}, 5.92 ; \mathrm{N}, 6.33$. Found: C, 62.44; H, 6.20; N, 6.48. 
Z-12; minor product, could not be isolated; $\mathrm{R}_{f}=0.15$ (EtOAc/petroleum ether $1 / 3$ ).

\section{Methyl $\left(1 R^{*}, 2 S^{*}\right)$ - and $\left(1 R^{*}, 2 R^{*}\right)-1$-(acetylamino)-2-(4-methoxyphenyl)-1-} cyclopropanecarboxylate 13 (Table 2 , entry 4).

$\boldsymbol{E - 1 3}$; colourless solid characterised from an enriched mixture of both diastereoisomers; EtOAc/petroleum ether $3: 2, \mathrm{R}_{f}=0.15 ;{ }^{1} \mathrm{H}$ NMR $\left(400 \mathrm{MHz}, \mathrm{CDCl}_{3}\right) \delta 1.58(1 \mathrm{H}, \mathrm{dd}, J=9.8$ and 5.9 $\left.\mathrm{Hz}, \mathrm{CHHCCO}_{2}\right), 2.04\left(3 \mathrm{H}, \mathrm{s}, \mathrm{CH}_{3} \mathrm{CONH}\right), 2.21\left(1 \mathrm{H}, \mathrm{dd}, J=8.8\right.$ and $\left.5.9 \mathrm{~Hz}, \mathrm{CH} H C C O_{2}\right), 2.77$ $(1 \mathrm{H}, \mathrm{dd}, J=9.8$ and $8.8 \mathrm{~Hz}, \mathrm{ArCH}), 3.37\left(3 \mathrm{H}, \mathrm{s}, \mathrm{COOCH}_{3}\right), 3.78(3 \mathrm{H}, \mathrm{s}, \mathrm{ArOCH}), 6.40(1 \mathrm{H}$, br. s, $\mathrm{NH}), 6.81(2 \mathrm{H}, \mathrm{d}, J=8.8 \mathrm{~Hz}$, Aryl H$), 7.26(2 \mathrm{H}, \mathrm{d}, J=8.8 \mathrm{~Hz}, \operatorname{Aryl~H}) ;{ }^{13} \mathrm{C}$ NMR $(100 \mathrm{MHz}$, $\left.\mathrm{CDCl}_{3}\right) \delta 20.7$ (t), 23.3 (q), 34.3 (d), 40.6 (s), 52.0 (q), 55.2 (q) 113.5 (2d), 127.3 (s), 130.3 (2d), 158.7 (s), 170.1 (s), 171.2 (s); GC-MS retention time $15.25 \mathrm{~min} ., \mathrm{m} / z$ (EI) $263\left(\mathrm{M}^{+}, 6\right), 231$ (38), 220 (64), 189 (90), 160 (100), 145 (67), 134 (34), 91 (36).

Z-13; colourless solid characterised from an enriched mixture of both diastereoisomers; EtOAc/petroleum ether 3:2, $\mathrm{R}_{f}=0.20 ;{ }^{1} \mathrm{H}$ NMR $\left(400 \mathrm{MHz}, \mathrm{CDCl}_{3}\right) \delta 1.66(1 \mathrm{H}, \mathrm{dd}, J=7.9$ and 5.9 $\mathrm{Hz}, \mathrm{CHH}), 1.85\left(3 \mathrm{H}, \mathrm{s}, \mathrm{CH}_{3} \mathrm{CONH}\right), 2.19(1 \mathrm{H}, \mathrm{dd}, J=9.5$ and $5.9 \mathrm{~Hz}, \mathrm{CH} H), 2.90(1 \mathrm{H}, \mathrm{dd}, J=9.5$ and $7.9 \mathrm{~Hz}, \mathrm{ArCH}), 3.74\left(3 \mathrm{H}, \mathrm{s}, \mathrm{COOCH}_{3}\right), 3.81\left(3 \mathrm{H}, \mathrm{s}, \mathrm{ArOCH}_{3}\right), 5.34(1 \mathrm{H}, \mathrm{br} . \mathrm{s}, \mathrm{NH}), 6.87(2 \mathrm{H}$, $\mathrm{d}, J=8.8 \mathrm{~Hz}, \operatorname{Aryl~H}), 7.09\left(2 \mathrm{H}, \mathrm{d}, J=8.8 \mathrm{~Hz}\right.$, Aryl H); ${ }^{13} \mathrm{C} \mathrm{NMR}\left(100 \mathrm{MHz}, \mathrm{CDCl}_{3}\right) \delta 21.3(\mathrm{t})$, 23.0 (q), 32.0 (d), 38.9 (s), 52.7 (q), 55.4 (q) 114.0 (2d), 126.1 (s), 129.8 (2d), 159.1 (s), 171.3 (s), 172.2 (s); IR $v_{\max } / \mathrm{cm}^{-1} 3280,1717,1661,1545,1517,1437,1337,1264,1247,1156,1029,838$, 682; MS m/z (EI) 263 (M+1 12), 231 (45), 220 (85), 189 (78), 160 (100), 145 (42), 91 (40); HRMS: found 263.1155, $\mathrm{C}_{14} \mathrm{H}_{17} \mathrm{NO}_{4}$ requires 263.1158.

$\left(1 R^{*}, \quad 2 S^{*}\right)-\quad$ and $\quad\left(1 R^{*}, \quad 2 R^{*}\right)-4-N i t r o b e n z y l \quad 1-[(t e r t-b u t o x y c a r b o n y l) a m i n o]-2-(4-$ methylphenyl)-1-cyclopropanecarboxylate 14 (Table 2, entry 5).

$\boldsymbol{E - 1 4}$; colourless solid; eluent petroleum ether/EtOAc 3:1, $\mathrm{R}_{f}=0.35$; m.p. $146-147{ }^{\circ} \mathrm{C}$ (EtOAc/petroleum ether); ${ }^{1} \mathrm{H}$ NMR $\left(400 \mathrm{MHz}, \mathrm{CDCl}_{3}\right) \delta 1.47\left(9 \mathrm{H}, \mathrm{s}, \mathrm{C}\left(\mathrm{CH}_{3}\right)_{3}\right), 1.63(1 \mathrm{H}, \mathrm{dd}, J=$ 9.2 and $\left.5.5 \mathrm{~Hz}, \mathrm{CHHCCO}_{2}\right), 2.19\left(1 \mathrm{H}, \mathrm{dd}, J=8.4\right.$ and $\left.5.5 \mathrm{~Hz}, \mathrm{C} H \mathrm{HCCO}_{2}\right), 2.21\left(3 \mathrm{H}, \mathrm{s}, \mathrm{ArCH}_{3}\right)$, $2.87(1 \mathrm{H}, \mathrm{dd}, J=9.2$ and $8.4 \mathrm{~Hz}, \mathrm{ArCH}), 4.91$ and $4.96\left(2 \mathrm{H}, \mathrm{AB}\right.$ System, $J_{\mathrm{AB}}=13.6 \mathrm{~Hz}$, $\left.\mathrm{COOCH}_{2}\right), 5.46(1 \mathrm{H}$, br. s, $\mathrm{NH}), 7.02(2 \mathrm{H}, \mathrm{d}, J=8.2 \mathrm{~Hz}, \operatorname{Aryl~H}), 7.08(2 \mathrm{H}, \mathrm{d}, J=8.2 \mathrm{~Hz}, \operatorname{Aryl~H})$, $7.19\left(2 \mathrm{H}\right.$, br. d, $J=8.8 \mathrm{~Hz}$, Aryl H), $8.05\left(2 \mathrm{H}, \mathrm{d}, J=8.8 \mathrm{~Hz}\right.$, Aryl H); ${ }^{13} \mathrm{C} \mathrm{NMR}\left(100 \mathrm{MHz}, \mathrm{CDCl}_{3}\right)$ $\delta 21.1(\mathrm{q}), 21.1(\mathrm{t}), 28.4(3 \mathrm{q}), 35.4(\mathrm{~d}), 41.2(\mathrm{~s}), 65.3(\mathrm{t}), 80.3(\mathrm{~s}), 123.4(2 \mathrm{~d}), 128.2$ (2d), 128.8 (2d), 129.3 (2d), 132.1 (s), 136.8 (s), 142.9 (s), 147.5 (s), 155.9 (s) 170.0 (s); IR v max $/ \mathrm{cm}^{-1} 3361$, 1730, 1708, 1605, 1508, 1447, 1346, 1249, 1152, 1062, 932, 825, 735; MS m/z (EI) 427 ([MH] ${ }^{+}$, 30), 371 (48), 327 (26), 234 (40), 190 (100), 173 (58), 144 (40), 137 (28), 117 (36), 90 (22), 78 
(24); HRMS: found [M-Boc] ${ }^{+}$325.1188, $\mathrm{C}_{18} \mathrm{H}_{17} \mathrm{~N}_{2} \mathrm{O}_{4}$ requires 325.1188. Anal. Calc'd for $\mathrm{C}_{23} \mathrm{H}_{26} \mathrm{~N}_{2} \mathrm{O}_{6}: \mathrm{C}, 64.78 ; \mathrm{H}, 6.15 ; \mathrm{N}, 6.57$. Found: $\mathrm{C}, 64.71 ; \mathrm{H}, 6.33 ; \mathrm{N}, 6.69$.

Z-14; minor product, could not be isolated; eluent petroleum ether/EtOAc 3:1, $\mathrm{R}_{f}=0.25$.

Methyl $\quad\left(1 R^{*}, \quad 2 S^{*}\right)-\quad$ and $\quad\left(1 R^{*}, \quad 2 R^{*}\right)$-1-(acetylamino)-2-(4-methylphenyl)-1cyclopropanecarboxylate 15 (Table 2, entry 6).

$\boldsymbol{E}-15$; colourless solid characterised from an enriched mixture of both diastereoisomers; EtOAc/petroleum ether 2:1, $\mathrm{R}_{f}=0.20 ;{ }^{1} \mathrm{H} \mathrm{NMR}\left(400 \mathrm{MHz}, \mathrm{CDCl}_{3}\right) \delta 1.59(1 \mathrm{H}, \mathrm{dd}, J=9.5$ and 5.7 $\left.\mathrm{Hz}, \mathrm{CHHCCO}_{2}\right), 2.05\left(3 \mathrm{H}, \mathrm{s}, \mathrm{CH}_{3} \mathrm{CONH}\right), 2.23\left(1 \mathrm{H}, \mathrm{dd}, J=8.4\right.$ and $\left.5.7 \mathrm{~Hz}, \mathrm{CH} H \mathrm{CCO}_{2}\right), 2.31(3 \mathrm{H}$, s, $\left.\mathrm{ArCH}_{3}\right), 2.78(1 \mathrm{H}, \mathrm{dd}, J=9.5$ and $8.4 \mathrm{~Hz}, \mathrm{ArCH}), 3.36\left(3 \mathrm{H}, \mathrm{s}, \mathrm{COOCH}_{3}\right), 6.31(1 \mathrm{H}, \mathrm{br} . \mathrm{s}, \mathrm{NH})$, $7.08\left(2 \mathrm{H}, \mathrm{d}, J=8.1 \mathrm{~Hz}\right.$, Aryl H), $7.22\left(2 \mathrm{H}, \mathrm{d}, J=8.1 \mathrm{~Hz}\right.$, Aryl H)$;{ }^{13} \mathrm{C} \mathrm{NMR}\left(100 \mathrm{MHz}, \mathrm{CDCl}_{3}\right) \delta$ $20.6(\mathrm{t}), 21.2$ (q), 23.4 (q), 34.6 (d), 40.7 (s), 52.0 (q), 128.8 (2d), 129.1 (2d), 132.1 (s), 136.7 (s), 170.1 (s), 171.1 (s).

Z-15; colourless solid; EtOAc/petroleum ether 2:1, $\mathrm{R}_{f}=0.25$; m.p. 105-107 ${ }^{\circ} \mathrm{C}$ (EtOAc/petroleum ether); ${ }^{1} \mathrm{H} \mathrm{NMR}\left(400 \mathrm{MHz}, \mathrm{CDCl}_{3}\right) \delta 1.70\left(1 \mathrm{H}, \mathrm{dd}, J=8.0\right.$ and $\left.5.9 \mathrm{~Hz}, \mathrm{CHCCO}_{2}\right), 1.85(3 \mathrm{H}, \mathrm{s}$, $\left.\mathrm{CH}_{3} \mathrm{CONH}\right), 2.21\left(1 \mathrm{H}, \mathrm{dd}, J=9.5\right.$ and $\left.5.9 \mathrm{~Hz}, \mathrm{CH} \mathrm{CCO}_{2}\right), 2.35\left(3 \mathrm{H}, \mathrm{s}, \mathrm{ArCH}_{3}\right), 2.90(1 \mathrm{H}, \mathrm{dd}, J=$ 9.5 and $8.0 \mathrm{~Hz}, \mathrm{ArCH}), 3.74\left(3 \mathrm{H}, \mathrm{s}, \mathrm{COOCH}_{3}\right), 5.32(1 \mathrm{H}$, br. s, $\mathrm{NH}), 7.05(2 \mathrm{H}, \mathrm{d}, J=7.9 \mathrm{~Hz}$, Aryl $\mathrm{H}), 7.14(2 \mathrm{H}, \mathrm{d}, J=7.9 \mathrm{~Hz}, \operatorname{Aryl~H}) ;{ }^{13} \mathrm{C} \mathrm{NMR}\left(68 \mathrm{MHz}, \mathrm{CDCl}_{3}\right) \delta 21.1$ (q), $21.3(\mathrm{t}), 23.0$ (q), 32.3 (d), 38.9 (s), 52.7 (q), 128.6 (2d), 129.3 (2d), 131.1 (s), 137.3 (s), 171.3 (s), 172.2 (s); IR $v_{\max } / \mathrm{cm}^{-1}$ $3317,1720,1661,1527,1439,1368,1335,1271,1206,1167,832,689 ; \mathrm{MS} \mathrm{m} / \mathrm{z}$ (EI) $247\left(\mathrm{M}^{+}\right.$, 35), 215 (45), 204 (87), 173 (76), 144 (100), 117 (76), 91 (76); HRMS: found 247.1208, $\mathrm{C}_{14} \mathrm{H}_{17} \mathrm{NO}_{3}$ requires 247.1208. Anal. Calc'd for $\mathrm{C}_{14} \mathrm{H}_{17} \mathrm{NO}_{3}$ : C, 68.00; H, 6.93; N, 5.66. Found: C, 68.30; H, 7.30; N, 5.73.

$\left(1 R^{*}, 2 S^{*}\right)$ - and $\left(1 R^{*}, 2 R^{*}\right)$-4-Nitrobenzyl 1-[(tert-butoxycarbonyl)amino]-2-(4-fluorophenyl)1-cyclopropanecarboxylate 16 (Table 2, entry 7).

$\boldsymbol{E}$-16; colourless crystal; eluent petroleum ether/EtOAc $3: 1, \mathrm{R}_{f}=0.35 ;$ m.p. $103-104{ }^{\circ} \mathrm{C}$ (EtOAc/petroleum ether); ${ }^{1} \mathrm{H} \mathrm{NMR}\left(400 \mathrm{MHz}, \mathrm{CDCl}_{3}\right) \delta 1.47\left(9 \mathrm{H}, \mathrm{s}, \mathrm{C}\left(\mathrm{CH}_{3}\right)_{3}\right), 1.66(1 \mathrm{H}, \mathrm{dd}, J=$ 9.8 and $\left.5.6 \mathrm{~Hz}, \mathrm{CH} H C C O_{2}\right), 2.15\left(1 \mathrm{H}, \mathrm{dd}, J=8.3\right.$ and $\left.5.6 \mathrm{~Hz}, \mathrm{CHHCCO}_{2}\right), 2.90(1 \mathrm{H}, \mathrm{dd}, J=9.8$ and $8.3 \mathrm{~Hz}, \mathrm{ArCH}), 4.92$ and $4.98\left(2 \mathrm{H}, \mathrm{AB}\right.$ System, $\left.J_{\mathrm{AB}}=13.2 \mathrm{~Hz}, \mathrm{COOCH}_{2}\right), 5.45(1 \mathrm{H}$, br. s, $\mathrm{NH}), 6.89\left(2 \mathrm{H}, \mathrm{dd}, J_{\mathrm{H}-\mathrm{F}}=8.8 \mathrm{~Hz}\right.$ and $J=8.3 \mathrm{~Hz}$, Aryl H), $7.19(2 \mathrm{H}, \mathrm{d}, J=8.3 \mathrm{~Hz}$, Aryl H), 7.32 $\left(2 \mathrm{H}\right.$, br. d, $J=8.8 \mathrm{~Hz}$, Aryl H), $8.10\left(2 \mathrm{H}, \mathrm{d}, J=8.8 \mathrm{~Hz}\right.$, Aryl H); ${ }^{13} \mathrm{C}$ NMR $\left(100 \mathrm{MHz}, \mathrm{CDCl}_{3}\right) \delta$ $21.3(\mathrm{t}), 28.4(3 \mathrm{q}), 35.1(\mathrm{~d}), 41.0(\mathrm{~s}), 65.4(\mathrm{t}), 80.4(\mathrm{~s}), 115.0\left(2 \mathrm{~d}, J_{\mathrm{C}-\mathrm{F}}=21.4 \mathrm{~Hz}\right), 123.6(2 \mathrm{~d})$, $128.3(2 \mathrm{~d}), 128.7(\mathrm{~s}), 131.0\left(2 \mathrm{~d}, J_{\mathrm{C}-\mathrm{F}}=7.7 \mathrm{~Hz}\right), 142.8(\mathrm{~s}), 147.6(\mathrm{~s}), 155.9(\mathrm{~s}), 162.0\left(\mathrm{~s}, J_{\mathrm{C}-\mathrm{F}}=\right.$ 
$244.7 \mathrm{~Hz}), 169.8$ (s); IR $v_{\max } / \mathrm{cm}^{-1} 3390,1714,1604,1510,1445,1346,1250,1233,1150,1112$, 1060, 922, 836, 771, 735; MS m/z (EI) 431 (M+34), 375 (90), 238 (32), 194 (100), 57 (92). Anal. Calc'd for $\mathrm{C}_{22} \mathrm{H}_{23} \mathrm{FN}_{2} \mathrm{O}_{6}: \mathrm{C}, 61.39 ; \mathrm{H}, 5.39 ; \mathrm{N}, 6.51$. Found: C, 61.56; H, 5.74; N, 6.74.

Z-16; colourless crystals; eluent petroleum ether/EtOAc 3:1, $\mathrm{R}_{f}=0.25 ;{ }^{1} \mathrm{H}$ NMR (400 MHz, $\left.\mathrm{CDCl}_{3}\right) \delta 1.31\left(9 \mathrm{H}, \mathrm{s}, \mathrm{C}\left(\mathrm{CH}_{3}\right)_{3}\right), 1.71\left(1 \mathrm{H}, \mathrm{m}, \mathrm{CHHCCO}_{2}\right), 2.12\left(1 \mathrm{H}, \mathrm{m}, \mathrm{CH} \mathrm{HCCO}_{2}\right), 3.04(1 \mathrm{H}, \mathrm{m}$, $\mathrm{ArCH}), 4.73\left(1 \mathrm{H}\right.$, br. s, NH), 4.92 and $4.98\left(2 \mathrm{H}, \mathrm{AB}\right.$ System, $\left.J_{\mathrm{AB}}=13.6 \mathrm{~Hz}, \mathrm{COOCH}_{2}\right), 7.01(2 \mathrm{H}$, $\mathrm{dd}, J_{\mathrm{H}-\mathrm{F}}=8.8 \mathrm{~Hz}$ and $J=8.4 \mathrm{~Hz}$, Aryl H), $7.19(2 \mathrm{H}, \mathrm{d}, J=8.4 \mathrm{~Hz}$, Aryl H), $7.54(2 \mathrm{H}, \mathrm{d}, J=8.1$ $\mathrm{Hz}, \operatorname{Aryl~H}), 8.21\left(2 \mathrm{H}, \mathrm{d}, J=8.1 \mathrm{~Hz}\right.$, Aryl H); ${ }^{13} \mathrm{C}$ NMR (100 MHz, $\left.\mathrm{CDCl}_{3}\right) \delta 22.0(\mathrm{t}), 28.1(3 \mathrm{q})$, $32.6(\mathrm{~d}), 41.1(\mathrm{~s}), 65.8(\mathrm{t}), 80.3(\mathrm{~s}), 115.2\left(2 \mathrm{~d}, J_{\mathrm{C}-\mathrm{F}}=20.8 \mathrm{~Hz}\right), 123.8(2 \mathrm{~d}), 128.1(2 \mathrm{~d}), 128.4(\mathrm{~s})$, $130.4\left(2 \mathrm{~d}, J_{\mathrm{C}-\mathrm{F}}=7.7 \mathrm{~Hz}\right), 143.1(\mathrm{~s}), 147.8(\mathrm{~s}), 155.6(\mathrm{~s}), 162.2\left(\mathrm{~s}, J_{\mathrm{C}-\mathrm{F}}=246.0 \mathrm{~Hz}\right), 172.1(\mathrm{~s})$.

\section{Methyl $\left(1 R^{*}, 2 S^{*}\right)$ - and $\left(1 R^{*}, 2 R^{*}\right)$-1-(acetylamino)-2-(4-fluorophenyl)-1-} cyclopropanecarboxylate 17 (Table 2, entry 8).

$\boldsymbol{E}-17$; colourless solid characterised from an enriched mixture of both diastereoisomers; EtOAc/petroleum ether 3:1, $\mathrm{R}_{f}=0.15 ;{ }^{1} \mathrm{H}$ NMR $\left(400 \mathrm{MHz}, \mathrm{CDCl}_{3}\right) \delta 1.60(1 \mathrm{H}, \mathrm{dd}, J=9.8$ and 5.8 $\left.\mathrm{Hz}, \mathrm{CHHCCO}_{2}\right), 2.06\left(3 \mathrm{H}, \mathrm{s}, \mathrm{CH}_{3} \mathrm{CONH}\right), 2.18\left(1 \mathrm{H}, \mathrm{dd}, J=8.3\right.$ and $\left.5.8 \mathrm{~Hz}, \mathrm{CH} H \mathrm{CCO}_{2}\right), 2.81(1 \mathrm{H}$, dd, $J=9.8$ and $8.3 \mathrm{~Hz}, \mathrm{ArCH}), 3.37\left(3 \mathrm{H}, \mathrm{s}, \mathrm{COOCH}_{3}\right), 6.38\left(1 \mathrm{H}\right.$, br. s, NH), $6.97\left(2 \mathrm{H}, \mathrm{dd}, J_{\mathrm{H}-\mathrm{F}}=\right.$ $8.8 \mathrm{~Hz}$ and $J=7.8 \mathrm{~Hz}$, Aryl H), $7.35\left(2 \mathrm{H}, \mathrm{dd}, J=7.8 \mathrm{~Hz}\right.$ and $J_{\mathrm{H}-\mathrm{F}}=5.4 \mathrm{~Hz}$, Aryl $\left.\mathrm{H}\right) ;{ }^{13} \mathrm{C} \mathrm{NMR}$ $\left(100 \mathrm{MHz}, \mathrm{CDCl}_{3}\right) \delta 20.8(\mathrm{t}), 23.3(\mathrm{q}), 34.3$ (d), 40.5 (s), 52.1 (q), 114.9 (2d, $\left.J_{\mathrm{C}-\mathrm{F}}=21.4 \mathrm{~Hz}\right)$, $130.9\left(2 \mathrm{~d}, J_{\mathrm{C}-\mathrm{F}}=8.4 \mathrm{~Hz}\right), 131.1\left(\mathrm{~s}, J_{\mathrm{C}-\mathrm{F}}=3.1 \mathrm{~Hz}\right), 162.0\left(\mathrm{~s}, J_{\mathrm{C}-\mathrm{F}}=245.9 \mathrm{~Hz}\right), 170.8(\mathrm{~s}), 171.3(\mathrm{~s})$.

Z-17; colourless solid; EtOAc/petroleum ether 3:1, $\mathrm{R}_{f}=0.25$; m.p. 143-145 ${ }^{\circ} \mathrm{C}$ (EtOAc/petroleum ether); ${ }^{1} \mathrm{H} \mathrm{NMR}\left(400 \mathrm{MHz}, \mathrm{CDCl}_{3}\right) \delta 1.68\left(1 \mathrm{H}, \mathrm{dd}, J=8.1\right.$ and $\left.5.9 \mathrm{~Hz}, \mathrm{CHHCCO}_{2}\right), 1.82(3 \mathrm{H}, \mathrm{s}$, $\left.\mathrm{CH}_{3} \mathrm{CONH}\right), 2.13\left(1 \mathrm{H}, \mathrm{dd}, J=9.3\right.$ and $\left.5.9 \mathrm{~Hz}, \mathrm{CH} H C C O_{2}\right), 2.97(1 \mathrm{H}, \mathrm{dd}, J=9.3$ and $8.1 \mathrm{~Hz}$, $\mathrm{ArCH}), 3.74\left(3 \mathrm{H}, \mathrm{s}, \mathrm{COOCH}_{3}\right), 5.71(1 \mathrm{H}$, br. s, $\mathrm{N} H), 7.01\left(2 \mathrm{H}, \mathrm{dd}, J=8.8 \mathrm{~Hz}\right.$ and $J_{\mathrm{H}-\mathrm{F}}=6.8 \mathrm{~Hz}$, Aryl H), $7.15\left(2 \mathrm{H}, \mathrm{dd}, J=8.8 \mathrm{~Hz}\right.$ and $J_{\mathrm{H}-\mathrm{F}}=5.7 \mathrm{~Hz}$, Aryl H); ${ }^{13} \mathrm{C} \mathrm{NMR}\left(100 \mathrm{MHz}, \mathrm{CDCl}_{3}\right) \delta 21.2$ (t), $22.8(\mathrm{q}), 31.7(\mathrm{~d}), 39.2(\mathrm{~s}), 52.7(\mathrm{q}), 115.3\left(2 \mathrm{~d}, J_{\mathrm{C}-\mathrm{F}}=21.4 \mathrm{~Hz}\right), 130.2\left(\mathrm{~s}, J_{\mathrm{C}-\mathrm{F}}=2.3 \mathrm{~Hz}\right), 130.3$ $\left(2 \mathrm{~d}, J_{\mathrm{C}-\mathrm{F}}=7.6 \mathrm{~Hz}\right), 162.2\left(\mathrm{~s}, J_{\mathrm{C}-\mathrm{F}}=245.5 \mathrm{~Hz}\right), 171.3(\mathrm{~s}), 172.0(\mathrm{~s}) ; \mathrm{IR} v_{\max } / \mathrm{cm}^{-1} 3326,1725,1667$, 1513, 1438, 1371, 1333, 1265, 1161, 1066, 982, 844, 824, 761; MS m/z (EI) $251\left(\mathrm{M}^{+}, 34\right), 233$ (36), 219 (47), 208 (81), 177 (42), 148 (100), 122 (39), 84 (23); HRMS: found 251.0950, $\mathrm{C}_{13} \mathrm{H}_{14} \mathrm{FNO}_{3}$ requires 251.0958. Anal. Calc'd for $\mathrm{C}_{13} \mathrm{H}_{14} \mathrm{FNO}_{3}: \mathrm{C}, 61.90 ; \mathrm{H}, 5.90 ; \mathrm{N}, 5.49$. Found: C, $62.14 ; \mathrm{H}, 5.62 ; \mathrm{N}, 5.57$.

$\left(1 R^{*}, \quad 2 S^{*}\right)-\quad$ and $\left(1 R^{*}, \quad 2 R^{*}\right)-4-N i t r o b e n z y l \quad 1-[($ tert-butoxycarbonyl)amino]-2-(2,2diphenylvinyl)-1-cyclopropanecarboxylate 18 (Table 2, entry 9). 
$\boldsymbol{E}$-18; yellow solid; eluent petroleum ether/EtOAc 3:1; m.p. 146-148 ${ }^{\circ} \mathrm{C}$ (EtOAc/petroleum ether); ${ }^{1} \mathrm{H}$ NMR $\left(300 \mathrm{MHz}\right.$ at $\left.55{ }^{\circ} \mathrm{C}, \mathrm{CDCl}_{3}\right) \delta 1.41\left(9 \mathrm{H}, \mathrm{s}, \mathrm{C}\left(\mathrm{CH}_{3}\right)_{3}\right), 1.55(1 \mathrm{H}, \mathrm{dd}, J=9.3$ and $5.4 \mathrm{~Hz}$, $\left.\mathrm{CHHCCO}_{2}\right), 1.97\left(1 \mathrm{H}, \mathrm{dd}, J=8.1\right.$ and $\left.5.4 \mathrm{~Hz}, \mathrm{CHHCCO}_{2}\right), 2.14(1 \mathrm{H}$, dd, $J=9.3$ and $8.1 \mathrm{~Hz}$, $\left.\mathrm{Ph}_{2} \mathrm{C}=\mathrm{CHCH}\right), 5.00\left(1 \mathrm{H}\right.$, br. s, NH), 5.19 and $5.35\left(2 \mathrm{H}, \mathrm{AB}\right.$ System, $\left.J_{\mathrm{AB}}=13.3 \mathrm{~Hz}, \mathrm{COOCH}_{2}\right)$, $5.94\left(1 \mathrm{H}, \mathrm{d}, J=9.3 \mathrm{~Hz}, \mathrm{Ph}_{2} \mathrm{C}=\mathrm{CH}\right), 7.00-7.42(10 \mathrm{H}, \mathrm{m}$, Aryl H), 7.41-7.49 (2H, m, Aryl H), 8.00$8.08\left(2 \mathrm{H}, \mathrm{m}\right.$, Aryl H); ${ }^{13} \mathrm{C} \mathrm{NMR}\left(75 \mathrm{MHz}, \mathrm{CDCl}_{3}\right) \delta 21.6(\mathrm{~s}), 25.3(\mathrm{t}), 28.2(3 \mathrm{q}), 32.5(\mathrm{~d}), 65.6(\mathrm{t})$, 80.4 (s), 123.7 (2d), 124.1 (d), 127.2, 127.5, 127.5, 127.7, 127.9, 128.1, 128.4, 129.6, 130.1, 139.6, 141.6, 142.9, 145.2, 147.5, 147.9, 155.5 (s), 170.8 (s); IR $v_{\max } / \mathrm{cm}^{-1} 1731,1686,1607,1508$, 1160, 845, 768, 739, 705, 699; MS m/z (CI with $\left.\mathrm{CH}_{4}\right) 469$ ([MH-NO$\left.]^{+}, 1\right), 441$ (3), 415 (64), 398 (12), 335 (34), 234 (100), 167 (80), 122 (42), 57 (66); HRMS (CI, [MH-Boc+H] $]^{+}$) found: 415.1657, $\mathrm{C}_{25} \mathrm{H}_{23} \mathrm{~N}_{2} \mathrm{O}_{4}$ requires 415.1658 .

Z-18; yellow solid; eluent petroleum ether/EtOAc 3:1; m.p. 112-113 ${ }^{\circ} \mathrm{C}$ (EtOAc/petroleum ether); ${ }^{1} \mathrm{H}$ NMR $\left(300 \mathrm{MHz}, 5{ }^{\circ} \mathrm{C}, \mathrm{CDCl}_{3}\right) \delta 1.42\left(9 \mathrm{H}, \mathrm{s}, \mathrm{C}\left(\mathrm{CH}_{3}\right)_{3}\right), 1.42\left(1 \mathrm{H}, \mathrm{m}, \mathrm{CH} \mathrm{HCCO}_{2}\right), 1.98(1 \mathrm{H}$, $\mathrm{dd}, J=9.2$ and $\left.5.1 \mathrm{~Hz}, \mathrm{C} H \mathrm{HCCO}_{2}\right), 2.46\left(1 \mathrm{H}, \mathrm{q}, J=9.2 \mathrm{~Hz}, \mathrm{Ph}_{2} \mathrm{C}=\mathrm{CHCH}\right), 5.06(1 \mathrm{H}, \mathrm{br} . \mathrm{s}, \mathrm{NH})$, 5.10 and $5.26\left(2 \mathrm{H}, \mathrm{AB}\right.$ System, $\left.J_{\mathrm{AB}}=13.6 \mathrm{~Hz}, \mathrm{COOCH}_{2}\right), 5.69\left(1 \mathrm{H}, \mathrm{d}, J=9.2 \mathrm{~Hz}, \mathrm{Ph}_{2} \mathrm{CCHCH}\right)$, 7.15-7.35 (10H, m, Aryl H), 7.35-7.43 (2H, m, Aryl H), 8.11-8.19 (2H, m, Aryl H); ${ }^{13} \mathrm{C}$ NMR (100 $\left.\mathrm{MHz}, \mathrm{CDCl}_{3}\right) \delta 25.3(\mathrm{t}), 28.1(\mathrm{~s}), 28.3(3 \mathrm{q}), 30.1(\mathrm{~d}), 65.5(\mathrm{t}), 80.5(\mathrm{~s}), 123.8,124.4,127.6,127.6$, 127.6, 128.1, 128.4, 128.5, 130.0, 139.5, 142.1, 143.1, 147.7, 156.1, 171.7; IR $v_{\max } / \mathrm{cm}^{-1} 1727$, 1693, 1607, 1510, 1338, 1156, 844, 762, 737; MS m/z (CI with $\left.\mathrm{CH}_{4}\right) 469$ ([MH-NO $\left.]^{+}, 1\right), 441$ (3), 415 (64), 398 (10), 335 (28), 234 (100), 167 (84), 122 (52) and 57 (84); HRMS (CI, [MHBoc $+\mathrm{H}]^{+}$) Found: 415.1657, $\mathrm{C}_{25} \mathrm{H}_{23} \mathrm{~N}_{2} \mathrm{O}_{4}$ requires 415.1658 .

Methyl $\quad\left(1 R^{*}, \quad 2 S^{*}\right)-\quad$ and $\quad\left(1 R^{*}, \quad 2 R^{*}\right)-1-($ acetylamino)-2-(2,2-diphenylvinyl)-1cyclopropanecarboxylate 19 (Table 2, entry 10).

$\boldsymbol{E}$-19; green solid; EtOAc/petroleum ether 7:3; ${ }^{1} \mathrm{H} \mathrm{NMR}\left(400 \mathrm{MHz}, \mathrm{CDCl}_{3}\right) \delta 1.53(1 \mathrm{H}, \mathrm{dd}, J=9.1$ and $\left.5.3 \mathrm{~Hz}, \mathrm{CHHCCO}_{2}\right), 2.04\left(3 \mathrm{H}, \mathrm{s}, \mathrm{CH}_{3} \mathrm{CONH}\right), 2.04\left(1 \mathrm{H}, \mathrm{m}, \mathrm{CH} H \mathrm{CCO}_{2}\right), 2.11(1 \mathrm{H}, \mathrm{q}, J=9.1$ $\left.\mathrm{Hz}, \mathrm{Ph}_{2} \mathrm{C}=\mathrm{CHCH}\right), 3.74\left(3 \mathrm{H}, \mathrm{s}, \mathrm{OCH}_{3}\right), 6.02\left(1 \mathrm{H}, \mathrm{d}, J=9.1 \mathrm{~Hz}, \mathrm{Ph}_{2} \mathrm{C}=\mathrm{CH}\right), 6.05(1 \mathrm{H}$, br. s, $\mathrm{N} H)$, 7.18-7.44 (10H, m, Aryl H); ${ }^{13} \mathrm{C}$ NMR (100 MHz, $\left.\mathrm{CDCl}_{3}\right) \delta 21.1(\mathrm{q}), 23.1$ (t), 31.5 (d), 41.3 (s), 53.0 (q), 124.3 (d), 127.3 (d), 127.4 (d), 127.5 (d), 127.8 (d), 127.9 (d), 128.2 (d), 128.4 (d), 128.5 (d), 129.9 (d), 130.3 (d), 140.0 (s), 141.9 (s), 145.0 (s), 170.8 (s), 172.1 (s).

Z-19; pale green solid; EtOAc/petroleum ether 7:3; m.p. 71-74 ${ }^{\circ} \mathrm{C}$ (EtOAc/petroleum ether); ${ }^{1} \mathrm{H}$ NMR (400 MHz, $\left.\mathrm{CDCl}_{3}\right) \delta 1.33\left(1 \mathrm{H}, \mathrm{dd}, J=7.5\right.$ and $\left.5.4 \mathrm{~Hz}, \mathrm{CH} \mathrm{HCCO}_{2}\right), 2.04\left(3 \mathrm{H}, \mathrm{s}, \mathrm{COCH}_{3}\right)$, $2.04\left(1 \mathrm{H}, \mathrm{dd}, J=9.3\right.$ and $\left.5.4 \mathrm{~Hz}, \mathrm{CH}_{H C C O}\right), 2.40\left(1 \mathrm{H}, \mathrm{td}, J=9.3\right.$ and $\left.7.5 \mathrm{~Hz}, \mathrm{Ph}_{2} \mathrm{C}=\mathrm{CHCH}\right)$, $3.64\left(3 \mathrm{H}, \mathrm{s}, \mathrm{OCH}_{3}\right), 5.60\left(1 \mathrm{H}, \mathrm{d}, J=9.3 \mathrm{~Hz}, \mathrm{Ph}_{2} \mathrm{C}=\mathrm{CHCH}\right), 6.10(1 \mathrm{H}$, br. s, NH) and 7.18-7.44 
(10H, m, Aryl H); ${ }^{13} \mathrm{C}$ NMR (100 MHz, $\left.\mathrm{CDCl}_{3}\right) \delta 23.3$ (q), 24.7 (t), 29.4 (d), 40.1 (s), 52.6 (q), 124.4 (d), 127.7 (2d), 127.7 (2d), 128.3 (2d), 128.4 (2d), 130.1 (2d), 139.4 (s), 142.1 (s), 146.4 (s), $171.5(\mathrm{~s}), 171.8(\mathrm{~s}) ; \mathrm{IR} \mathrm{v}_{\max } / \mathrm{cm}^{-1} 1729,1662,1532,1266,1195,1155,765,699$; MS m/z (CI with $\left.\mathrm{CH}_{4}\right) 336\left([(\mathrm{MH})]^{+}, 100\right)$; Anal. Calc'd for $\mathrm{C}_{21} \mathrm{H}_{21} \mathrm{NO}_{3}$ : C, 75.2; H, 6.3; N, 4.2. Found: C, 75.2; $\mathrm{H}, 6.4 ; \mathrm{N}, 4.3$.

$\left(1 R^{*}, 2 S^{*}\right)$ - and $\left(1 R^{*}, 2 R^{*}\right)-4-N i t r o b e n z y l ~ 1-[(t e r t-b u t o x y c a r b o n y l) a m i n o]-2-(3-[1-(t e r t-b u t y l)-$ 1,1-dimethylsilyl]oxyphenyl)-1-cyclopropanecarboxylate 20 (Table 2, entry 11).

See experimental section in paper.

Methyl $\quad\left(1 R^{*}, \quad 2 S^{*}\right)-\quad$ and $\quad\left(1 R^{*}, \quad 2 R^{*}\right)-1-($ acetylamino)-2-(3-[1-(tert-butyl)-1,1dimethylsilyl]oxyphenyl)-1-cyclopropanecarboxylate 21 (Table 2, entry 12).

$\boldsymbol{E}$-21; colourless oil (7\% yield); EtOAc/petroleum ether $1: 1, \mathrm{R}_{f}=0.15 ;{ }^{1} \mathrm{H} \mathrm{NMR}(400 \mathrm{MHz}$, $\left.\mathrm{CDCl}_{3}\right) \delta 0.18\left(6 \mathrm{H}, \mathrm{s}, \mathrm{Si}\left(\mathrm{CH}_{3}\right)_{2}\right), 0.97\left(9 \mathrm{H}, \mathrm{s}, \mathrm{SiC}\left(\mathrm{CH}_{3}\right)_{3}\right), 1.59(1 \mathrm{H}, \mathrm{dd}, J=9.9$ and $5.5 \mathrm{~Hz}$, $\left.\mathrm{CHHCCO}_{2}\right), 2.05\left(3 \mathrm{H}, \mathrm{s}, \mathrm{CH}_{3} \mathrm{CONH}\right), 2.21\left(1 \mathrm{H}, \mathrm{dd}, J=8.4\right.$ and $\left.5.5 \mathrm{~Hz}, \mathrm{CH} H \mathrm{CCO}_{2}\right), 2.77(1 \mathrm{H}, \mathrm{dd}$, $J=9.9$ and $8.4 \mathrm{~Hz}, \mathrm{ArCH}), 3.34\left(3 \mathrm{H}, \mathrm{s}, \mathrm{COOCH}_{3}\right), 6.33(1 \mathrm{H}$, br. s, $\mathrm{NH}), 6.73(1 \mathrm{H}, \mathrm{dd}, J=8.1 \mathrm{~Hz}$ and $1.8 \mathrm{~Hz}$, Aryl H), $6.83(1 \mathrm{H}, \mathrm{d}, J=1.8 \mathrm{~Hz}$, Aryl H) $6.92(1 \mathrm{H}, \mathrm{d}, J=7.7 \mathrm{~Hz}, \operatorname{Aryl~H}), 7.12(1 \mathrm{H}$, dd, $J=8.1 \mathrm{~Hz}$ and $7.7 \mathrm{~Hz}$, Aryl H); ${ }^{13} \mathrm{C}$ NMR (100 MHz, $\left.\mathrm{CDCl}_{3}\right) \delta-4.3(2 \mathrm{q}), 18.3$ (s) 20.5 (t), 23.3 (q), 25.8 (3q), 34.4 (d), 40.7 (s), 51.9 (q), 118.9 (d), 121.1 (d), 122.3 (d), 129.0 (d), 136.8 (s), 155.5 (s), 170.0 (s), 171.1 (s); IR $v_{\max } / \mathrm{cm}^{-1} 3289,2931,2858,1737,1662,1532,1487,1436,1334$, 1277, 1151, 1005, 918, 833, 779, 693; MS m/z (EI) 363 (M+1, 63), 306 (92), 245 (100), 232 (100), 204 (90); HRMS: found 363.1854, $\mathrm{C}_{19} \mathrm{H}_{29} \mathrm{NO}_{4} \mathrm{Si}$ requires 363.1866.

Z-21; colourless solid (37\% yield); EtOAc/petroleum ether 1:1, $\mathrm{R}_{f}=0.25$; m.p. $110-112{ }^{\circ} \mathrm{C}$ (EtOAc/petroleum ether); ${ }^{1} \mathrm{H}$ NMR $\left(400 \mathrm{MHz}, \mathrm{CDCl}_{3}\right) \delta 0.19\left(6 \mathrm{H}, \mathrm{s}, \mathrm{Si}\left(\mathrm{CH}_{3}\right)_{2}\right), 0.98(9 \mathrm{H}, \mathrm{s}$, $\left.\mathrm{SiC}\left(\mathrm{CH}_{3}\right)_{3}\right), 1.68\left(1 \mathrm{H}, \mathrm{dd}, J=8.0\right.$ and $\left.6.0 \mathrm{~Hz}, \mathrm{CHHCCO}_{2}\right), 1.86\left(3 \mathrm{H}, \mathrm{s}, \mathrm{CH}_{3} \mathrm{CONH}\right), 2.22(1 \mathrm{H}$, dd, $J=9.5$ and $\left.6.0 \mathrm{~Hz}, \mathrm{CH} H \mathrm{CCO}_{2}\right), 2.88(1 \mathrm{H}, \mathrm{dd}, J=9.5$ and $8.0 \mathrm{~Hz}, \mathrm{ArCH}), 3.75\left(3 \mathrm{H}, \mathrm{s}, \mathrm{COOCH}_{3}\right)$, $5.31(1 \mathrm{H}$, br. s, NH), $6.63(1 \mathrm{H}, \mathrm{d}, J=8.1 \mathrm{~Hz}$, Aryl H), $6.77(2 \mathrm{H}, \mathrm{m}, \operatorname{Aryl} \mathrm{H}), 7.19(1 \mathrm{H}, \mathrm{t}, J=7.9$ $\mathrm{Hz}$, Aryl H); ${ }^{13} \mathrm{C}$ NMR (100 MHz, $\mathrm{CDCl}_{3}$ ) $\delta-4.3$ (2q), 18.2 (s) 21.3 (t), 22.9 (q), 25.7 (3q), 32.3 (d), 39.0 (s), 52.7 (q), 119.2 (d), 120.4 (d), 121.9 (d), 129.5 (d), 135.9 (s), 155.9 (s), 171.2 (s), 172.1 (s); IR $v_{\max } / \mathrm{cm}^{-1} 3299,2959,1742,1665,1602,1542,1489,1270,1249,1159,971,921$, 834, 781, 687; MS m/z (EI) $363\left(\mathrm{M}^{+}, 32\right), 304$ (100), 245 (72), 232 (71), 204 (70); HRMS: found 363.1862, $\mathrm{C}_{19} \mathrm{H}_{29} \mathrm{NO}_{4} \mathrm{Si}$ requires 363.1866. Anal. Calc'd for $\mathrm{C}_{19} \mathrm{H}_{29} \mathrm{NO}_{4} \mathrm{Si}$ : C, 62.78; H, 8.04; N, 3.85. Found: C, $62.63 \mathrm{H}, 8.39$; N, 4.00 . 
$\left(1 R^{*}, 2 S^{*}\right)-\quad$ and $\left(1 R^{*}, 2 R^{*}\right)-4-N i t r o b e n z y l \quad 1-[($ tert-butoxycarbonyl)amino]-2-vinyl-1cyclopropanecarboxylate 22 (Table 2 , entry 13).

$\boldsymbol{E}$-22; colourless solid (26\% yield); EtOAc/petrol 25:75, $R_{\mathrm{f}}=0.31$; m.p. $69-70{ }^{\circ} \mathrm{C}$ (EtOAc/petrol); ${ }^{1} \mathrm{H}$ NMR $\left(300 \mathrm{MHz}, \mathrm{CDCl}_{3}\right) \delta 1.41\left(9 \mathrm{H}, \mathrm{s}, \mathrm{C}\left(\mathrm{CH}_{3}\right)_{3}\right), 1.54(1 \mathrm{H}, \mathrm{dd}, J=8.7$ and $5.4 \mathrm{~Hz}$, $\left.\mathrm{CHHCCO}_{2}\right), 1.83\left(1 \mathrm{H}, \mathrm{dd}, J=8.7\right.$ and $\left.5.4 \mathrm{~Hz}, \mathrm{CH} \mathrm{HCCO}_{2}\right), 2.21\left(1 \mathrm{H}, \mathrm{q}, J=8.7 \mathrm{~Hz}, \mathrm{CH}_{2}=\mathrm{CHCH}\right)$, $5.11(1 \mathrm{H}, \mathrm{dd}, J=10.3$ and $1.7 \mathrm{~Hz}, \mathrm{CHH}=\mathrm{CH}), 5.22(1 \mathrm{H}$, br. s, $\mathrm{NH}), 5.22$ and $5.28(2 \mathrm{H}, \mathrm{AB}$ System, $\left.J_{A B}=13.5 \mathrm{~Hz}, \mathrm{CCO}_{2} \mathrm{CH} H \mathrm{Ar}\right), 5.29(1 \mathrm{H}, \mathrm{dd}, J=17.2$ and $1.7 \mathrm{~Hz}, \mathrm{CH} H=\mathrm{CH}), 5.74(1 \mathrm{H}$, ddd, $J=17.2,10.3$ and $\left.8.7 \mathrm{~Hz}, \mathrm{CH}_{2}=\mathrm{CH}\right), 7.46-7.54(2 \mathrm{H}, \mathrm{m}$, Aryl H), 8.14-8.22 (2H, m, Aryl H); ${ }^{13} \mathrm{C}$ NMR (100 MHz, $\mathrm{CDCl}_{3}$ ) $\delta 23.9(\mathrm{t}), 28.3(3 \mathrm{q}), 34.8(\mathrm{~d}), 40.8(\mathrm{~s}), 65.5(\mathrm{t}), 80.3(\mathrm{~s}), 118.2(\mathrm{t})$, 123.7 (2d), 128.1 (2d), 133.3 (d), 143.2 (s), 147.8 (s), 155.7 (s), 170.5 (s); IR v max $/ \mathrm{cm}^{-1}$ 1710, 1607, 1521, 1346, 1153, 994, 912, 845; MS m/z (ES) 363 ([MH] $\left.]^{+}, 41\right), 348$ (26), 307 (100), 263 (97), 136 (17); HRMS (ES, [MH-C( $\left.\left.\left(\mathrm{CH}_{3}\right)_{3}+\mathrm{H}\right]^{+}\right)$: found 307.0922. $\mathrm{C}_{14} \mathrm{H}_{15} \mathrm{~N}_{2} \mathrm{O}_{6}$ requires 307.0930.

Z-22; colourless solid (10\% yield); EtOAc/petrol 25:75, $R_{\mathrm{f}}=0.26 ;$ m.p. $117-119{ }^{\circ} \mathrm{C}$ (EtOAc/petrol); ${ }^{1} \mathrm{H}$ NMR $\left(300 \mathrm{MHz}\right.$ at $\left.55{ }^{\circ} \mathrm{C}, \mathrm{CDCl}_{3}\right) \delta 1.27(1 \mathrm{H}, \mathrm{dd}, J=8.4$ and $5.1 \mathrm{~Hz}$, $\left.\mathrm{CHHCCO}_{2}\right), 1.41\left(9 \mathrm{H}, \mathrm{s}, \mathrm{C}\left(\mathrm{CH}_{3}\right)_{3}\right), 1.92\left(1 \mathrm{H}, \mathrm{dd}, J=8.4\right.$ and $\left.5.1 \mathrm{~Hz}, \mathrm{CH} H \mathrm{CCO}_{2}\right), 2.43(1 \mathrm{H}, \mathrm{q}, J=$ $\left.8.4 \mathrm{~Hz}, \mathrm{CH}_{2}=\mathrm{CHCH}\right), 4.91(1 \mathrm{H}$, br. s, $\mathrm{NH}), 5.24(1 \mathrm{H}, \mathrm{d}, J=9.7 \mathrm{~Hz}, \mathrm{CHH}=\mathrm{CH}), 5.21$ and $5.27(2 \mathrm{H}$, AB System, $\left.J_{A B}=13.7 \mathrm{~Hz}, \mathrm{CCO}_{2} \mathrm{CH} H \mathrm{Ar}\right), 5.28(1 \mathrm{H}, \mathrm{d}, J=17.2 \mathrm{~Hz}, \mathrm{CH} H=\mathrm{CH}), 5.57(1 \mathrm{H}, \mathrm{ddd}, J=$ 17.2, 9.7 and $\left.8.4 \mathrm{~Hz}, \mathrm{CH}_{2}=\mathrm{CH}\right), 7.44-7.54\left(2 \mathrm{H}, \mathrm{m}\right.$, Aryl H), 8.15-8.23 (2H, m, Aryl H); ${ }^{13} \mathrm{C}$ NMR $\left(75 \mathrm{MHz}, \mathrm{CDCl}_{3}\right) \delta 23.6(\mathrm{t}), 28.3$ (3q), 31.9 (d), 39.4 (s), 65.7 (t), 80.4 (s), 118.9 (t), 123.9 (2d), 128.1 (2d), 133.4 (d), 143.1 (s), 147.8 (s), 156.0 (s), 172.2 (s); IR $v_{\max } / \mathrm{cm}^{-1}$ 1732, 1684, 1608, 1507, 1339, 1157, 913, 846; MS m/z (ES) 363 ([MH] , 35), 348 (17), 307 (89), 263 (100), 241 (6), 136 (15); HRMS (ES, [MH-C( $\left.\left.\left(\mathrm{CH}_{3}\right)_{3}+\mathrm{H}\right]^{+}\right)$; found 307.0922, $\mathrm{C}_{14} \mathrm{H}_{15} \mathrm{~N}_{2} \mathrm{O}_{6}$ requires 307.0930.

\section{X-ray Crystallographic Data.}

Methyl (1R*, 2R*)-1-(acetylamino)-2-(2,2-diphenylvinyl)-1-cyclopropanecarboxylate Z-19.

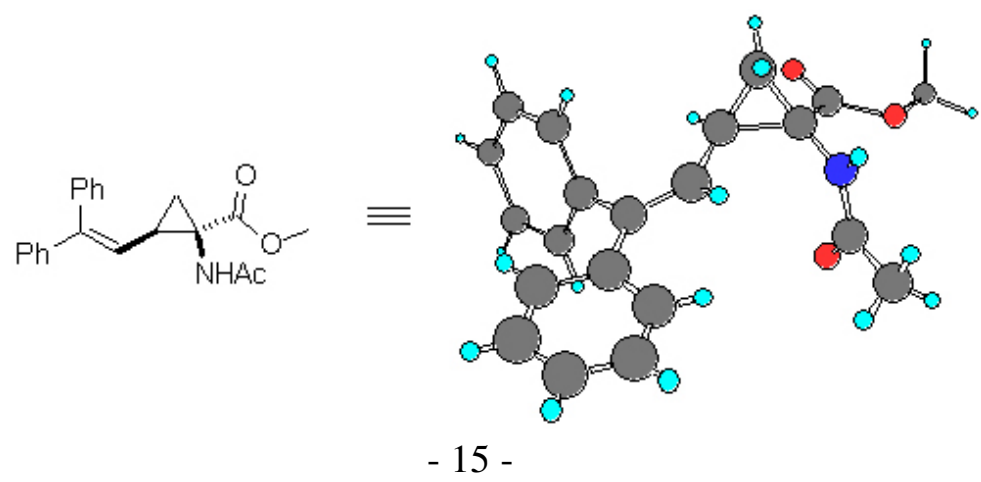


The cyclopropane crystallised from petroleum ether/EtOAc in the form of colourless blocks; crystallographic data is presented in Tables 4 and 5. A single crystal of the cyclopropane was coated in high-vacuum grease and mounted on a glass fibre. X-ray measurements were made using a Bruker SMART CCD area-detector diffractometer with Mo-K $\alpha$ radiation $(\lambda=0.71073 \AA) .{ }^{19}$ Intensities were integrated ${ }^{20}$ from several series of exposures, each exposure covering $0.3^{\circ}$ in $\omega$, and the total data set being a hemisphere. Absorption corrections were applied, based on multiple and symmetry-equivalent measurements. ${ }^{21}$ The structure was solved by direct methods and refined by least squares on weighted $\mathrm{F}^{2}$ values for all reflections (see Table 4). ${ }^{22}$ All non-hydrogen atoms were assigned anisotropic displacement parameters and refined without positional constraints. All hydrogen atoms were constrained to ideal geometries and refined with fixed isotropic displacement parameters 1.2 times that of their parent atoms. Refinement proceeded smoothly to give the residuals. Complex neutral-atom scattering factors were used. ${ }^{23}$

Table 4. Crystal data and structure refinement for Methyl (1R*, 2R*)-1-(acetylamino)-2-(2,2diphenylvinyl)-1-cyclopropanecarboxylate Z-19.

\begin{tabular}{lll} 
Empirical formula & $\mathrm{C}_{21} \mathrm{H}_{21} \mathrm{~N} \mathrm{O}_{3}$ \\
Formula weight & 335.39 & \\
Temperature & $173(2) \mathrm{K}$ \\
Wavelength & $0.71073 \AA$ \\
Crystal system & \multicolumn{1}{c}{ Monoclinic } \\
Space group & $\mathrm{P} 2(1) / \mathrm{n}$ & \\
Unit cell dimensions & $\mathrm{a}=9.6815(19) \AA \quad \alpha=90^{\circ}$ \\
& $\mathrm{b}=20.478(4) \AA \quad \quad \beta=118.67(3)^{\circ}$ \\
& $\mathrm{c}=9.968(2) \AA \gamma=90^{\circ}$
\end{tabular}

Volume $\quad 1733.9(6) \AA^{3}$

Z 4

Density (calculated) $1.285 \mathrm{Mg} / \mathrm{m}^{3}$

Absorption coefficient $\quad 0.086 \mathrm{~mm}^{-1}$

$\mathrm{F}(000)$

Crystal size $\quad 0.20 \times 0.20 \times 0.10 \mathrm{~mm}$

$\theta$ range for data collection 1.99 to $27.48^{\circ}$

Index ranges $\quad-11<=\mathrm{h}<=12,-20<=\mathrm{k}<=26,-12<=\mathrm{l}<=12$ 
Reflections collected 12125

Independent reflections $3966\left[\mathrm{R}_{\mathrm{int}}=0.0561\right]$

Completeness to $\theta=27.48^{\circ} \quad 99.8 \%$

Absorption correction Semi-empirical from equivalents

Max. and min. transmission 0.993 and 0.879

Refinement method Full-matrix least-squares on $\mathrm{F}^{2}$

Data / restraints / parameters 3966 / 0 / 231

Goodness-of-fit on $\mathrm{F}^{2} \quad \mathrm{~S}=0.948$

$\mathrm{R}$ indices [for 2441 reflections with $\mathrm{I}>2 \sigma(\mathrm{I})] \quad \mathrm{R}_{1}=0.0487, \mathrm{wR}_{2}=0.1012$

$\mathrm{R}$ indices (for all 3966 data) $\mathrm{R}_{1}=0.0916, \mathrm{wR}_{2}=0.1130$

Weighting scheme $\quad \mathrm{w}^{-1}=\sigma^{2}\left(\mathrm{~F}_{\mathrm{O}}^{2}\right)+(\mathrm{aP})^{2}$,

$$
\text { where } \mathrm{P}=\left[\max \left(\mathrm{F}_{\mathrm{o}}^{2}, 0\right)+2 \mathrm{~F}_{\mathrm{c}}^{2}\right] / 3
$$

$\mathrm{a}=0.0542$

Largest diff. peak and hole 0.278 and $-0.209 \mathrm{e}^{-3}$

Table 5. Bond lengths $[\AA]$ and angles $\left[{ }^{\circ}\right]$ for Methyl $\left(1 R^{*}, 2 R^{*}\right)-1-($ acetylamino)-2-(2,2diphenylvinyl)-1-cyclopropanecarboxylate Z-19.

$\begin{array}{ll}\mathrm{C}(1)-\mathrm{C}(2) & 1.385(3) \\ \mathrm{C}(1)-\mathrm{C}(6) & 1.394(2) \\ \mathrm{C}(1)-\mathrm{H}(1) & 0.9500 \\ \mathrm{C}(2)-\mathrm{C}(3) & 1.382(3) \\ \mathrm{C}(2)-\mathrm{H}(2) & 0.9500 \\ \mathrm{C}(3)-\mathrm{C}(4) & 1.377(3) \\ \mathrm{C}(3)-\mathrm{H}(3) & 0.9500 \\ \mathrm{C}(4)-\mathrm{C}(5) & 1.392(3) \\ \mathrm{C}(4)-\mathrm{H}(4) & 0.9500 \\ \mathrm{C}(5)-\mathrm{C}(6) & 1.390(2) \\ \mathrm{C}(5)-\mathrm{H}(5) & 0.9500 \\ \mathrm{C}(6)-\mathrm{C}(7) & 1.490(2) \\ \mathrm{C}(7)-\mathrm{C}(14) & 1.340(2) \\ \mathrm{C}(7)-\mathrm{C}(8) & 1.492(2) \\ \mathrm{C}(8)-\mathrm{C}(9) & 1.387(3) \\ \mathrm{C}(8)-\mathrm{C}(13) & 1.388(3) \\ \mathrm{C}(9)-\mathrm{C}(10) & 1.386(3)\end{array}$




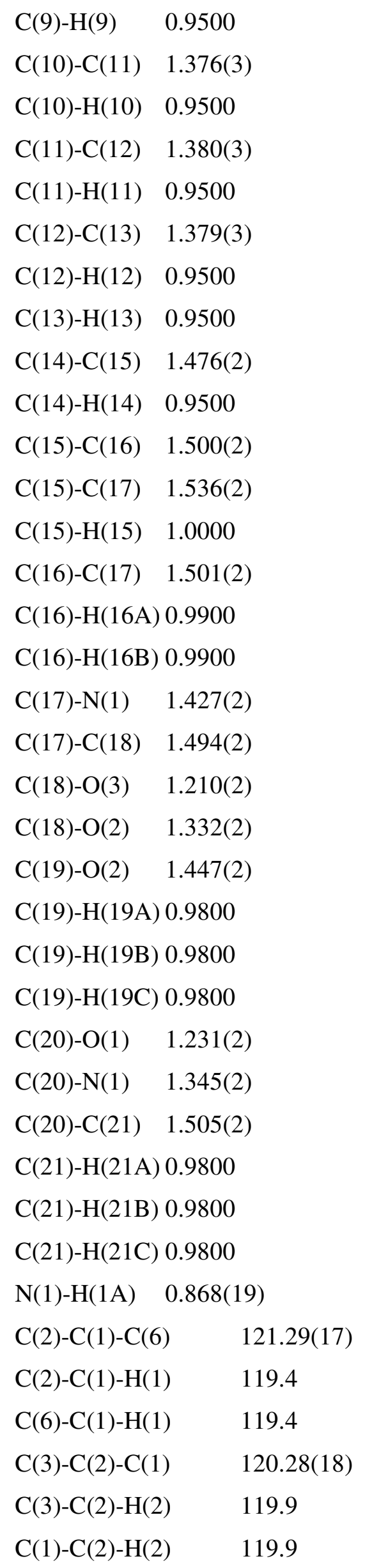




\begin{tabular}{|c|c|}
\hline $\mathrm{C}(4)-\mathrm{C}(3)-\mathrm{C}(2)$ & $119.39(18)$ \\
\hline $\mathrm{C}(4)-\mathrm{C}(3)-\mathrm{H}(3)$ & 120.3 \\
\hline $\mathrm{C}(2)-\mathrm{C}(3)-\mathrm{H}(3)$ & 120.3 \\
\hline$C(3)-C(4)-C(5)$ & $120.23(18)$ \\
\hline $\mathrm{C}(3)-\mathrm{C}(4)-\mathrm{H}(4)$ & 119.9 \\
\hline $\mathrm{C}(5)-\mathrm{C}(4)-\mathrm{H}(4)$ & 119.9 \\
\hline$C(6)-C(5)-C(4)$ & $121.26(17)$ \\
\hline $\mathrm{C}(6)-\mathrm{C}(5)-\mathrm{H}(5)$ & 119.4 \\
\hline $\mathrm{C}(4)-\mathrm{C}(5)-\mathrm{H}(5)$ & 119.4 \\
\hline$C(5)-C(6)-C(1)$ & $117.51(16)$ \\
\hline$C(5)-C(6)-C(7)$ & $121.05(15)$ \\
\hline $\mathrm{C}(1)-\mathrm{C}(6)-\mathrm{C}(7)$ & $121.44(15)$ \\
\hline$C(14)-C(7)-C(6)$ & $121.51(15)$ \\
\hline$C(14)-C(7)-C(8)$ & $120.76(16)$ \\
\hline $\mathrm{C}(6)-\mathrm{C}(7)-\mathrm{C}(8)$ & $117.70(14)$ \\
\hline $\mathrm{C}(9)-\mathrm{C}(8)-\mathrm{C}(13)$ & $118.54(17)$ \\
\hline $\mathrm{C}(9)-\mathrm{C}(8)-\mathrm{C}(7)$ & $121.59(17)$ \\
\hline $\mathrm{C}(13)-\mathrm{C}(8)-\mathrm{C}(7)$ & $119.87(17)$ \\
\hline$C(10)-C(9)-C(8)$ & $120.68(19)$ \\
\hline $\mathrm{C}(10)-\mathrm{C}(9)-\mathrm{H}(9)$ & 119.7 \\
\hline $\mathrm{C}(8)-\mathrm{C}(9)-\mathrm{H}(9)$ & 119.7 \\
\hline $\mathrm{C}(11)-\mathrm{C}(10)-\mathrm{C}(9)$ & $119.8(2)$ \\
\hline $\mathrm{C}(11)-\mathrm{C}(10)-\mathrm{H}(10)$ & 120.1 \\
\hline $\mathrm{C}(9)-\mathrm{C}(10)-\mathrm{H}(10)$ & 120.1 \\
\hline$C(10)-C(11)-C(12)$ & $120.18(19)$ \\
\hline $\mathrm{C}(10)-\mathrm{C}(11)-\mathrm{H}(11)$ & 119.9 \\
\hline $\mathrm{C}(12)-\mathrm{C}(11)-\mathrm{H}(11)$ & 119.9 \\
\hline$C(13)-C(12)-C(11)$ & $119.8(2)$ \\
\hline $\mathrm{C}(13)-\mathrm{C}(12)-\mathrm{H}(12)$ & 120.1 \\
\hline $\mathrm{C}(11)-\mathrm{C}(12)-\mathrm{H}(12)$ & 120.1 \\
\hline$C(12)-C(13)-C(8)$ & $120.9(2)$ \\
\hline $\mathrm{C}(12)-\mathrm{C}(13)-\mathrm{H}(13)$ & 119.5 \\
\hline $\mathrm{C}(8)-\mathrm{C}(13)-\mathrm{H}(13)$ & 119.5 \\
\hline$C(7)-C(14)-C(15)$ & $127.34(16)$ \\
\hline $\mathrm{C}(7)-\mathrm{C}(14)-\mathrm{H}(14)$ & 116.3 \\
\hline $\mathrm{C}(15)-\mathrm{C}(14)-\mathrm{H}(14)$ & 116.3 \\
\hline$C(14)-C(15)-C(16)$ & $118.92(15)$ \\
\hline
\end{tabular}




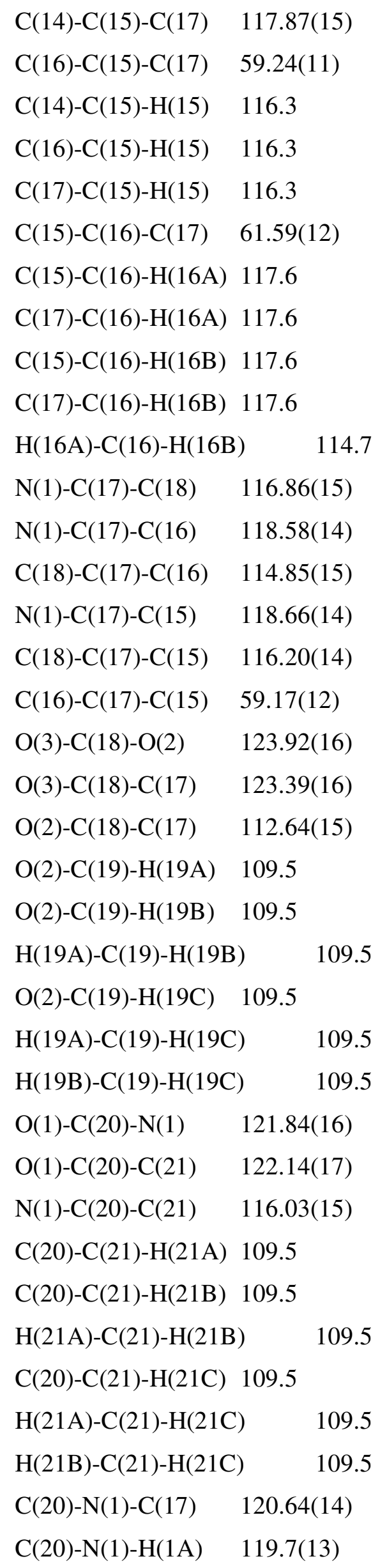


$\mathrm{C}(17)-\mathrm{N}(1)-\mathrm{H}(1 \mathrm{~A}) \quad 119.6(13)$

$\mathrm{C}(18)-\mathrm{O}(2)-\mathrm{C}(19) \quad 115.15(14)$ 
Acid.

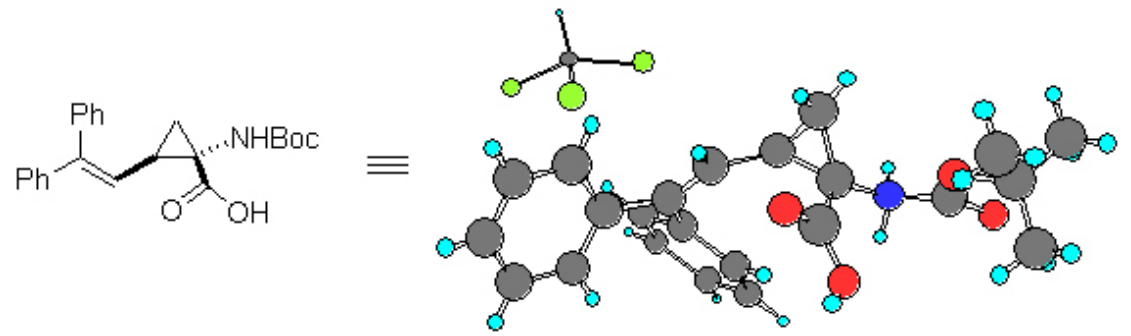

The cyclopropane crystallised from chloroform/pentane by vapour diffusion to give single crystals; crystallographic data is presented in Tables 6 and 7. A single crystal of the cyclopropane was coated in high-vacuum grease and mounted on a glass fibre. X-ray measurements were made using a Bruker SMART CCD area-detector diffractometer with Mo-K $\alpha$ radiation $(\lambda=0.71073 \AA$ ). Intensities were integrated from several series of exposures, each exposure covering $0.3^{\circ}$ in $\omega$, and the total data set being a hemisphere. Absorption corrections were applied, based on multiple and symmetry-equivalent measurements. The structure was solved by direct methods and refined by least squares on weighted $\mathrm{F}^{2}$ values for all reflections (see Table 6). All non-hydrogen atoms were assigned anisotropic displacement parameters and refined without positional constraints. All hydrogen atoms were constrained to ideal geometries and refined with fixed isotropic displacement parameters 1.2 times that of their parent atoms. Refinement proceeded smoothly to give the residuals shown in Table 00. Complex neutral-atom scattering factors were used.

Table 6. Crystal data and structure refinement for $\left(1 S^{*}, 2 R^{*}\right)-1-[($ tert-Butoxycarbonyl)amino]-2(2,2-diphenylvinyl)-1-cyclopropanecarboxylic Acid.

Empirical formula $\quad \mathrm{C}_{24} \mathrm{H}_{26} \mathrm{Cl}_{3} \mathrm{~N} \mathrm{O}_{4}$

Formula weight $\quad 498.81$

Temperature 173(2) K

Wavelength $0.71073 \AA$

Crystal system Monoclinic

Space group P2(1)/c

Unit cell dimensions $\mathrm{a}=12.425(3) \AA \quad \alpha=90^{\circ}$

$$
\begin{array}{ll}
\mathrm{b}=18.223(5) \AA & \beta=103.042(17)^{\circ} \\
\mathrm{c}=11.601(2) \AA & \gamma=90^{\circ}
\end{array}
$$

Volume $\quad 2558.9(11) \AA^{3}$ 
Z 4

Density (calculated) $1.295 \mathrm{Mg} / \mathrm{m}^{3}$

Absorption coefficient $\quad 0.387 \mathrm{~mm}^{-1}$

$\mathrm{F}(000) 1040$

Crystal size $0.2 \times 0.2 \times 0.05 \mathrm{~mm}$

$\theta$ range for data collection 2.02 to $24.01^{\circ}$

Index ranges $-14<=\mathrm{h}<=9,-20<=\mathrm{k}<=20,-13<=\mathrm{l}<=12$

Reflections collected 12277

Independent reflections $\quad 4000\left[\mathrm{R}_{\mathrm{int}}=0.0381\right]$

Completeness to $\theta=24.01^{\circ} \quad 99.7 \%$

Absorption correctionSemi-empirical from equivalents

Max. and min. transmission 0.981 and 0.926

Refinement method Full-matrix least-squares on $\mathrm{F}^{2}$

Data / restraints / parameters 4000 / 0 / 295

Goodness-of-fit on $\mathrm{F}^{2} \mathrm{~S}=1.067$

$\mathrm{R}$ indices [for 2905 reflections with $\mathrm{I}>2 \sigma(\mathrm{I})$ ] $\quad \mathrm{R}_{1}=0.0745, \mathrm{wR}_{2}=0.1994$

$\mathrm{R}$ indices (for all 4000 data) $\mathrm{R}_{1}=0.0991, \mathrm{wR}_{2}=0.2216$

Weighting scheme $\quad \mathrm{w}^{-1}=\sigma^{2}\left(\mathrm{~F}_{\mathrm{o}}^{2}\right)+(\mathrm{aP})^{2}$,

where $\mathrm{P}=\left[\max \left(\mathrm{F}_{\mathrm{o}}^{2}, 0\right)+2 \mathrm{~F}_{\mathrm{c}}^{2}\right] / 3$

$\mathrm{a}=0.1057$

Largest diff. peak and hole 1.435 and $-1.008 \mathrm{e}^{-3}$

Table 7. Bond lengths $[\AA]$ and angles $\left[{ }^{\circ}\right]$ for $\left(1 S^{*}, 2 R^{*}\right)-1-[($ tert-Butoxycarbonyl)amino]-2(2,2-diphenylvinyl)-1-cyclopropanecarboxylic Acid E-25.

$\begin{array}{ll}\mathrm{C}(1)-\mathrm{C}(2) & 1.383(5) \\ \mathrm{C}(1)-\mathrm{C}(6) & 1.389(5) \\ \mathrm{C}(1)-\mathrm{H}(1) & 0.9500 \\ \mathrm{C}(2)-\mathrm{C}(3) & 1.382(6) \\ \mathrm{C}(2)-\mathrm{H}(2 \mathrm{~A}) & 0.9500 \\ \mathrm{C}(3)-\mathrm{C}(4) & 1.384(6) \\ \mathrm{C}(3)-\mathrm{H}(3) & 0.9500 \\ \mathrm{C}(4)-\mathrm{C}(5) & 1.392(5) \\ \mathrm{C}(4)-\mathrm{H}(4) & 0.9500 \\ \mathrm{C}(5)-\mathrm{C}(6) & 1.397(5)\end{array}$




$$
\begin{aligned}
& \mathrm{C}(5)-\mathrm{H}(5) \quad 0.9500 \\
& \mathrm{C}(6)-\mathrm{C}(7) \quad \text { 1.493(5) } \\
& \text { C(7)-C(14) 1.338(5) } \\
& \mathrm{C}(7)-\mathrm{C}(8) \quad 1.495(5) \\
& \text { C(8)-C(13) 1.396(6) } \\
& \mathrm{C}(8)-\mathrm{C}(9) \quad 1.398(6) \\
& \text { C(9)-C(10) 1.395(6) } \\
& \mathrm{C}(9)-\mathrm{H}(9) \quad 0.9500 \\
& \text { C(10)-C(11) 1.370(7) } \\
& \text { C(10)-H(10) } 0.9500 \\
& \text { C(11)-C(12) 1.375(7) } \\
& \text { C(11)-H(11) } 0.9500 \\
& \text { C(12)-C(13) 1.388(6) } \\
& \text { C(12)-H(12) } 0.9500 \\
& \text { C(13)-H(13) } 0.9500 \\
& \text { C(14)-C(15) 1.487(5) } \\
& \text { C(14)-H(14) } 0.9500 \\
& \text { C(15)-C(16) 1.496(5) } \\
& \mathrm{C}(15)-\mathrm{C}(17) \quad 1.538(5) \\
& \mathrm{C}(15)-\mathrm{H}(15) \quad 1.0000 \\
& \mathrm{C}(16)-\mathrm{C}(17) \quad 1.505(5) \\
& \text { C(16)-H(16A) } 0.9900 \\
& \text { C(16)-H(16B) } 0.9900 \\
& \mathrm{C}(17)-\mathrm{N}(1) \quad 1.443(5) \\
& \text { C(17)-C(18) 1.499(5) } \\
& \mathrm{C}(18)-\mathrm{O}(1) \quad 1.211(4) \\
& \mathrm{C}(18)-\mathrm{O}(2) \quad 1.321(5) \\
& \mathrm{C}(19)-\mathrm{O}(3) \quad 1.227(5) \\
& \mathrm{C}(19)-\mathrm{O}(4) \quad 1.332(5) \\
& \mathrm{C}(19)-\mathrm{N}(1) \quad 1.343(5) \\
& \mathrm{C}(20)-\mathrm{O}(4) \quad 1.488(4) \\
& \mathrm{C}(20)-\mathrm{C}(22) \quad 1.509(7) \\
& \text { C(20)-C(23) 1.512(6) } \\
& \mathrm{C}(20)-\mathrm{C}(21) \quad 1.516(6) \\
& \text { C(21)-H(21A) } 0.9800 \\
& \mathrm{C}(21)-\mathrm{H}(21 \mathrm{~B}) 0.9800 \\
& \text { C(21)-H(21C) } 0.9800
\end{aligned}
$$




\begin{tabular}{|c|c|}
\hline $\mathrm{C}(22)-\mathrm{H}(22 \mathrm{~A}) 0.9$ & \\
\hline $\mathrm{C}(22)-\mathrm{H}(22 \mathrm{~B}) 0$. & \\
\hline $\mathrm{C}(22)-\mathrm{H}(22 \mathrm{C}) 0$ & \\
\hline $\mathrm{C}(23)-\mathrm{H}(23 \mathrm{~A}) 0$ & \\
\hline $\mathrm{C}(23)-\mathrm{H}(23 \mathrm{~B}) 0$ & \\
\hline $\mathrm{C}(23)-\mathrm{H}(23 \mathrm{C}) 0$ & \\
\hline $\mathrm{N}(1)-\mathrm{H}(1 \mathrm{~A})$ & \\
\hline $\mathrm{O}(2)-\mathrm{H}(2)$ & \\
\hline $\mathrm{C}(24)-\mathrm{Cl}(2)$ & (6) \\
\hline $\mathrm{C}(24)-\mathrm{Cl}(1)$ & \\
\hline $\mathrm{C}(24)-\mathrm{Cl}(3)$ & (5) \\
\hline $\mathrm{C}(24)-\mathrm{H}(24)$ & \\
\hline$C(2)-C(1)-C(6)$ & $121.3(4)$ \\
\hline $\mathrm{C}(2)-\mathrm{C}(1)-\mathrm{H}(1)$ & 119.3 \\
\hline $\mathrm{C}(6)-\mathrm{C}(1)-\mathrm{H}(1)$ & 119.3 \\
\hline$C(3)-C(2)-C(1)$ & $120.2(4)$ \\
\hline $\mathrm{C}(3)-\mathrm{C}(2)-\mathrm{H}(2 \mathrm{~A})$ & 119.9 \\
\hline $\mathrm{C}(1)-\mathrm{C}(2)-\mathrm{H}(2 \mathrm{~A})$ & 119.9 \\
\hline$C(2)-C(3)-C(4)$ & $119.5(4)$ \\
\hline $\mathrm{C}(2)-\mathrm{C}(3)-\mathrm{H}(3)$ & 120.2 \\
\hline $\mathrm{C}(4)-\mathrm{C}(3)-\mathrm{H}(3)$ & 120.2 \\
\hline$C(3)-C(4)-C(5)$ & $120.4(4)$ \\
\hline $\mathrm{C}(3)-\mathrm{C}(4)-\mathrm{H}(4)$ & 119.8 \\
\hline $\mathrm{C}(5)-\mathrm{C}(4)-\mathrm{H}(4)$ & 119.8 \\
\hline$C(4)-C(5)-C(6)$ & $120.4(4)$ \\
\hline $\mathrm{C}(4)-\mathrm{C}(5)-\mathrm{H}(5)$ & 119.8 \\
\hline $\mathrm{C}(6)-\mathrm{C}(5)-\mathrm{H}(5)$ & 119.8 \\
\hline$C(1)-C(6)-C(5)$ & $118.2(3)$ \\
\hline$C(1)-C(6)-C(7)$ & $120.3(3)$ \\
\hline$C(5)-C(6)-C(7)$ & $121.4(3)$ \\
\hline$C(14)-C(7)-C(6)$ & $120.1(3)$ \\
\hline $\mathrm{C}(14)-\mathrm{C}(7)-\mathrm{C}(8)$ & $122.9(3)$ \\
\hline $\mathrm{C}(6)-\mathrm{C}(7)-\mathrm{C}(8)$ & $116.9(3)$ \\
\hline $\mathrm{C}(13)-\mathrm{C}(8)-\mathrm{C}(9)$ & $118.4(4)$ \\
\hline$C(13)-C(8)-C(7)$ & $120.6(3)$ \\
\hline $\mathrm{C}(9)-\mathrm{C}(8)-\mathrm{C}(7)$ & $120.9(3)$ \\
\hline $\mathrm{C}(10)-\mathrm{C}(9)-\mathrm{C}(8)$ & $120.4(4)$ \\
\hline
\end{tabular}




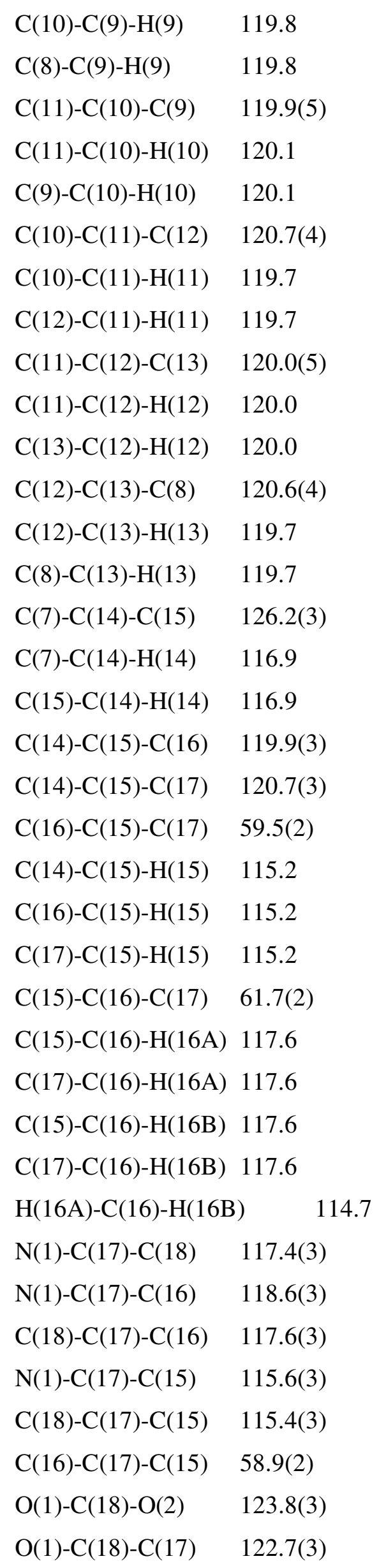




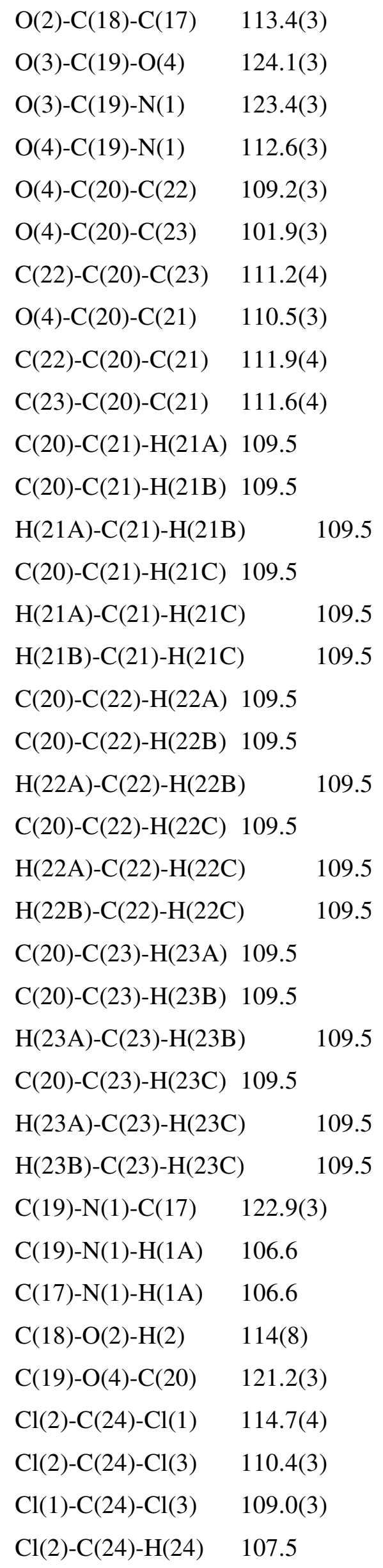


$\mathrm{Cl}(1)-\mathrm{C}(24)-\mathrm{H}(24) \quad 107.5$

$\mathrm{Cl}(3)-\mathrm{C}(24)-\mathrm{H}(24) \quad 107.5$

8. Spectra of new compounds. 


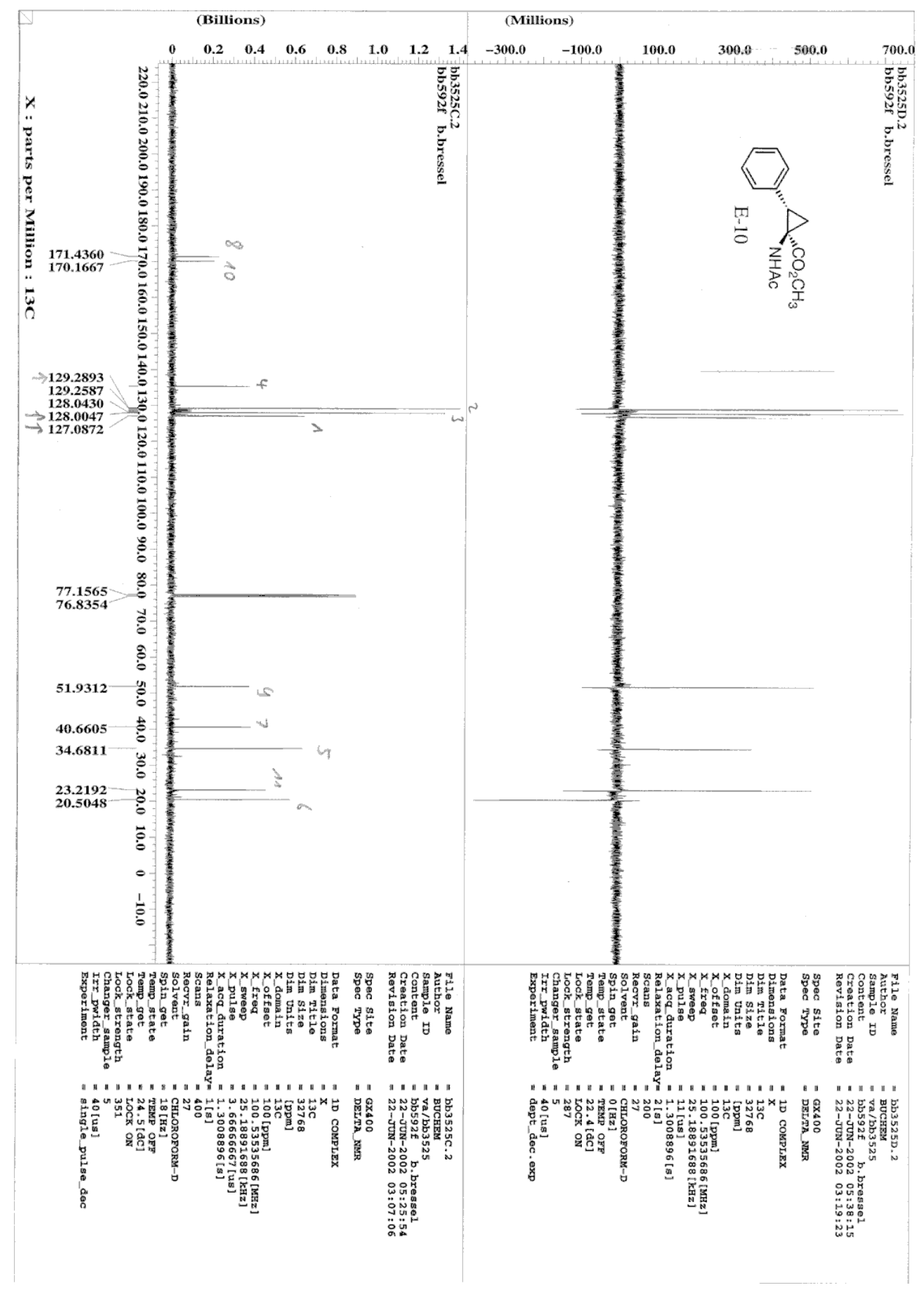




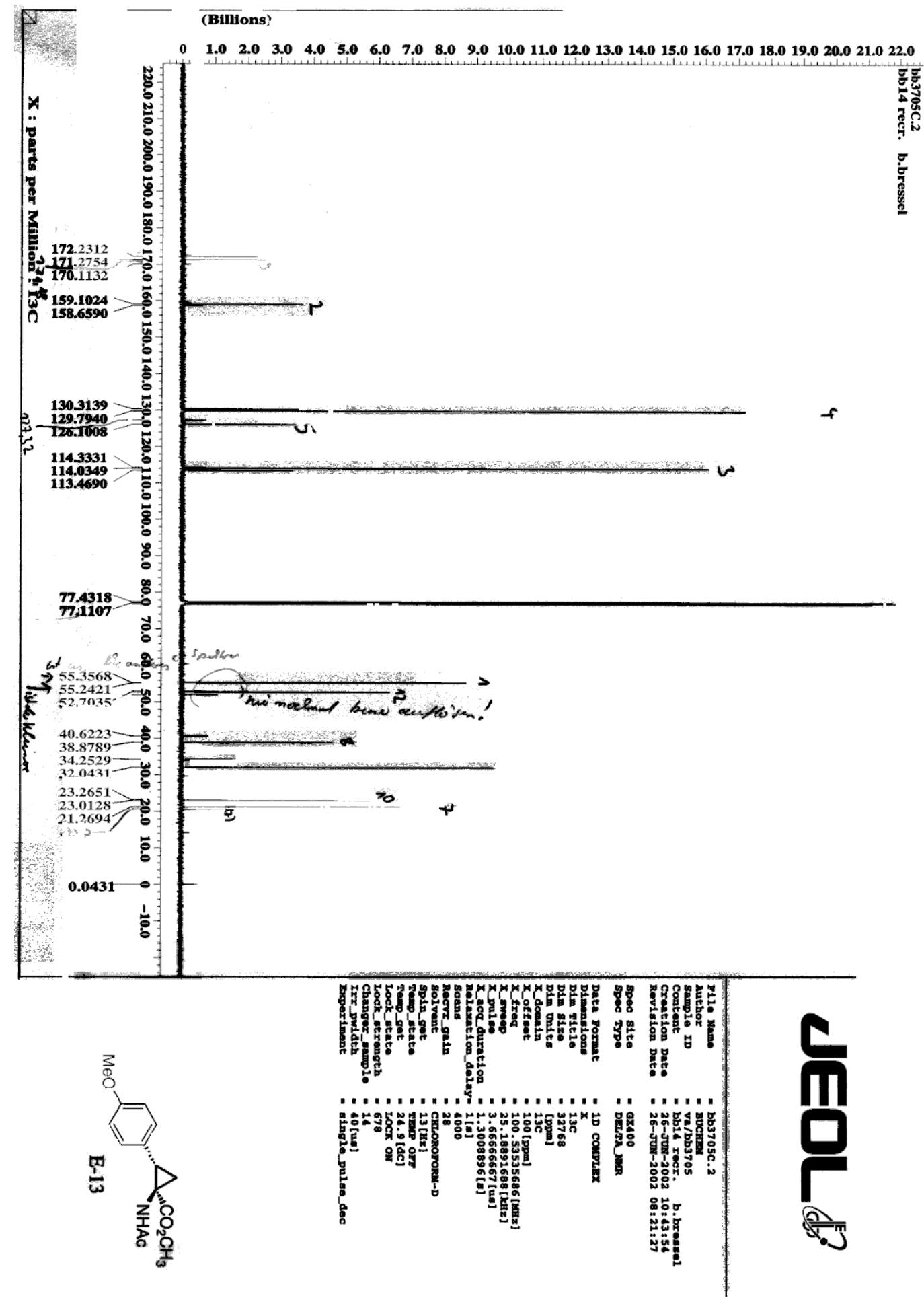




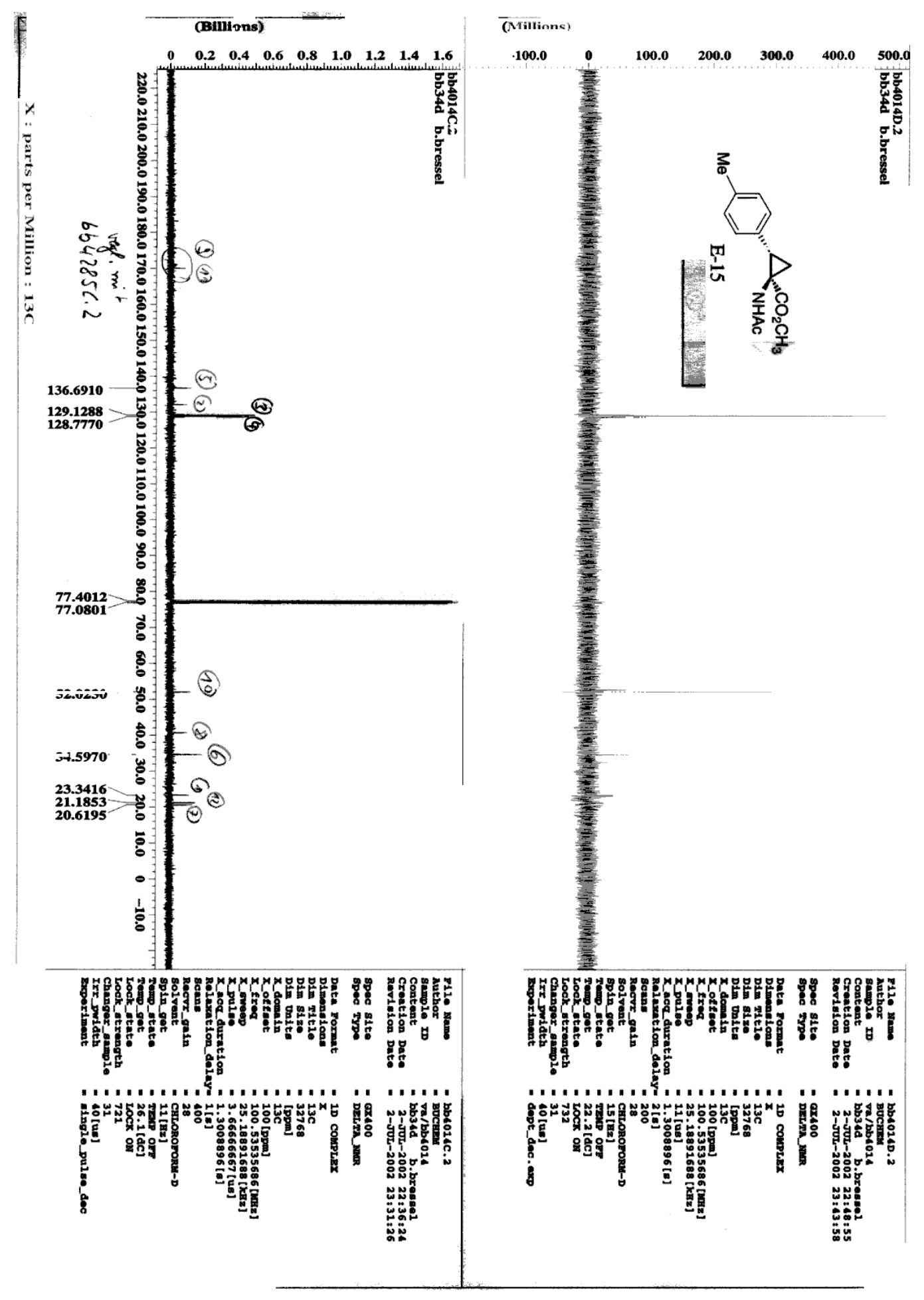




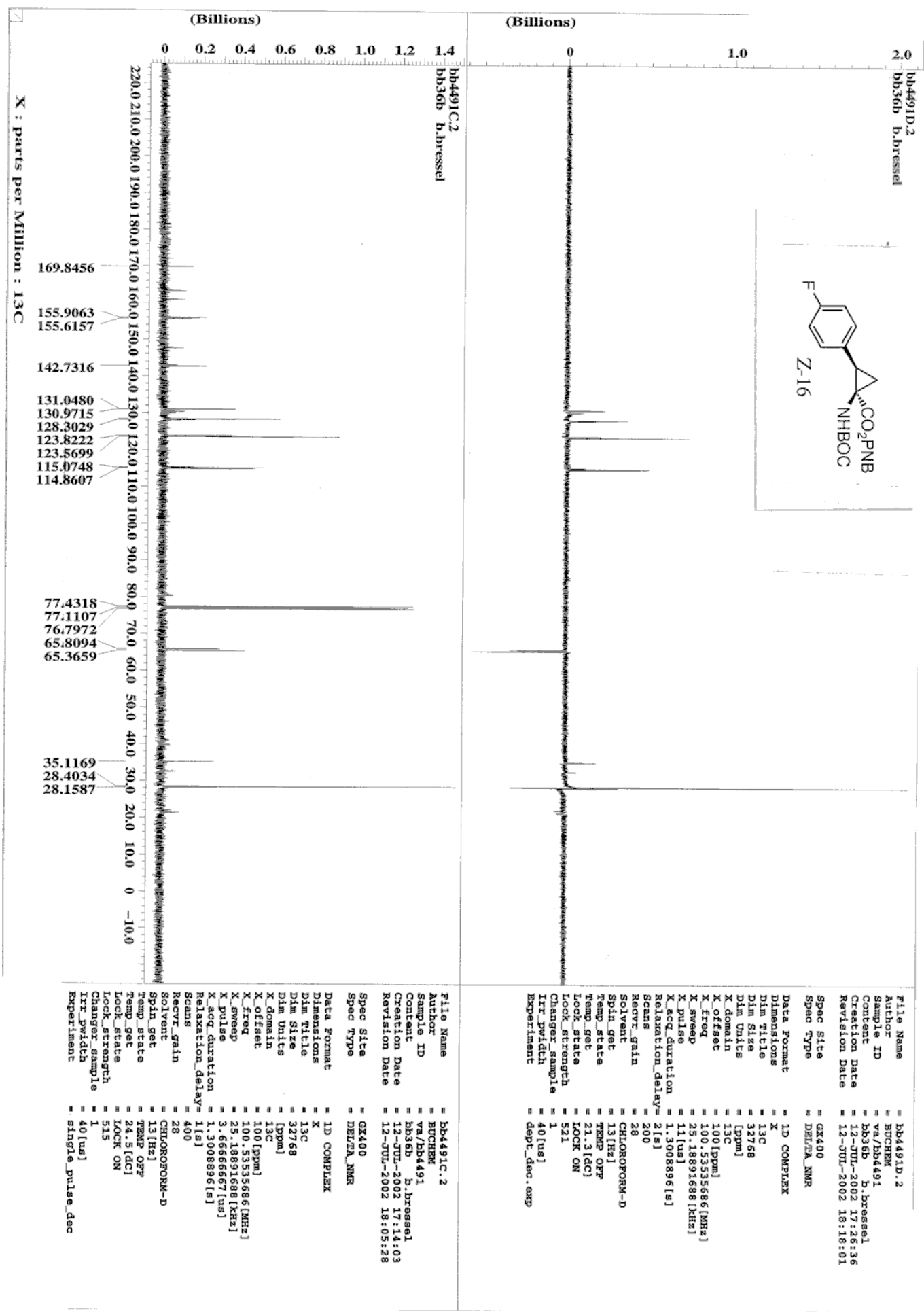




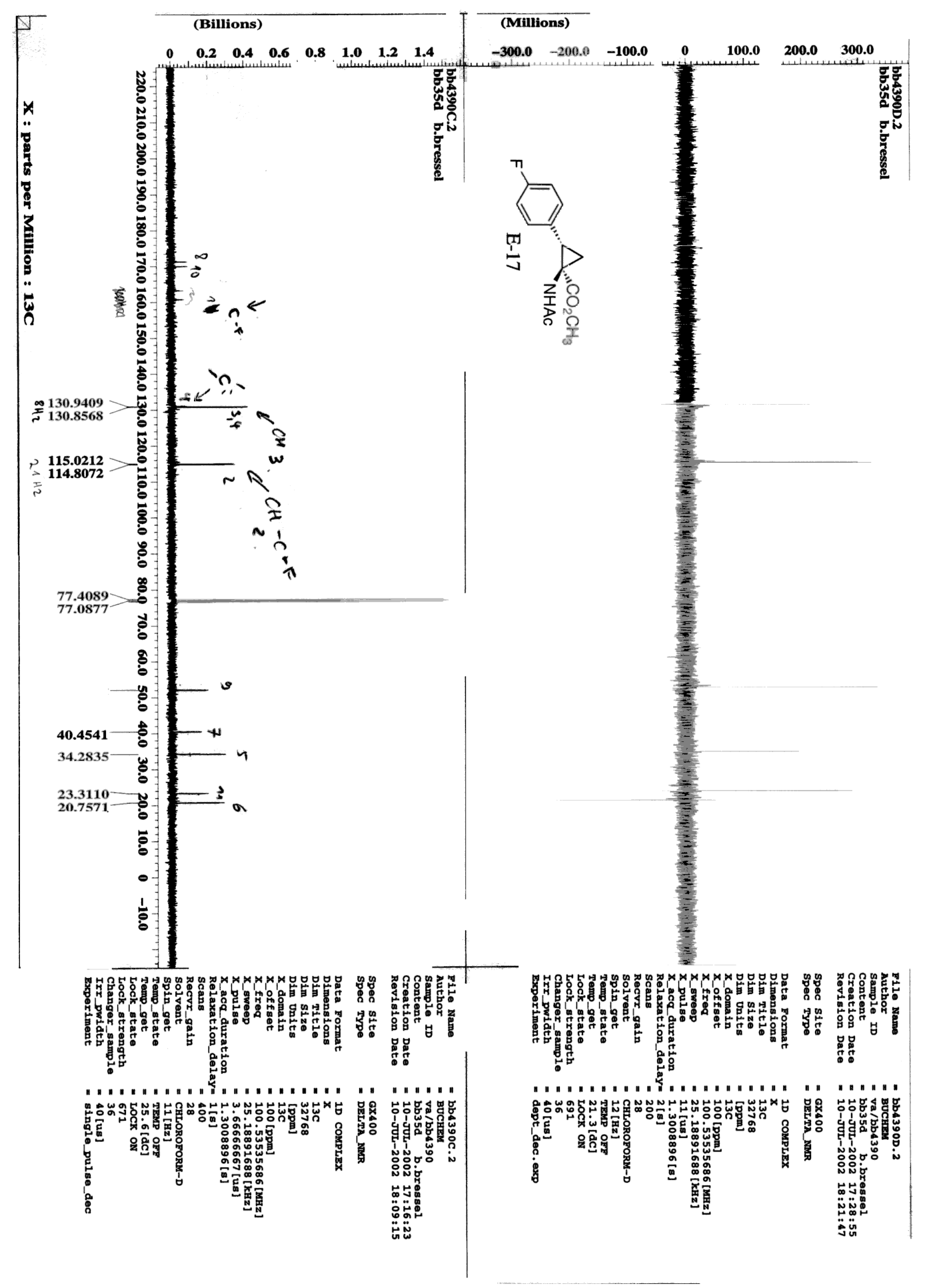




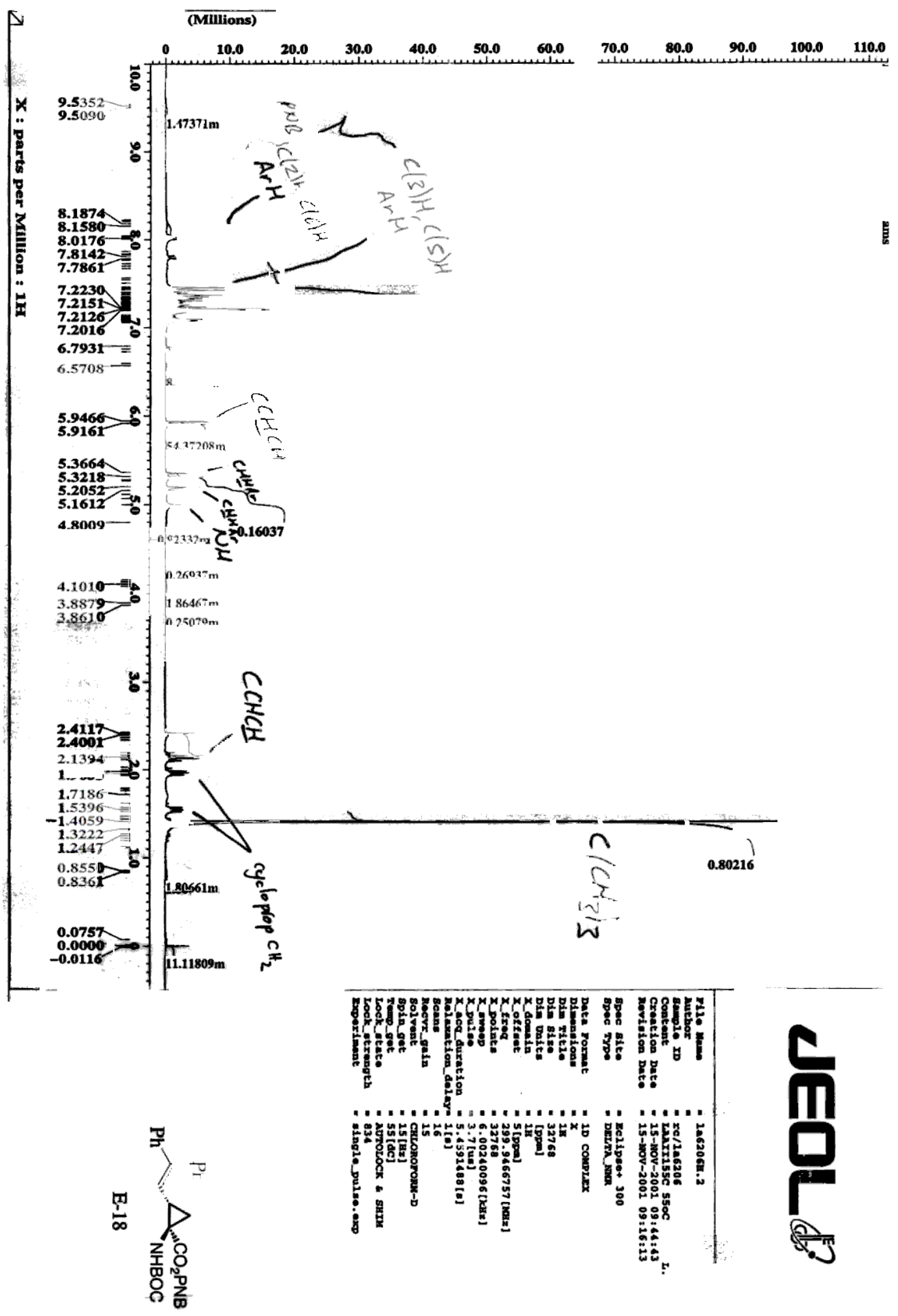




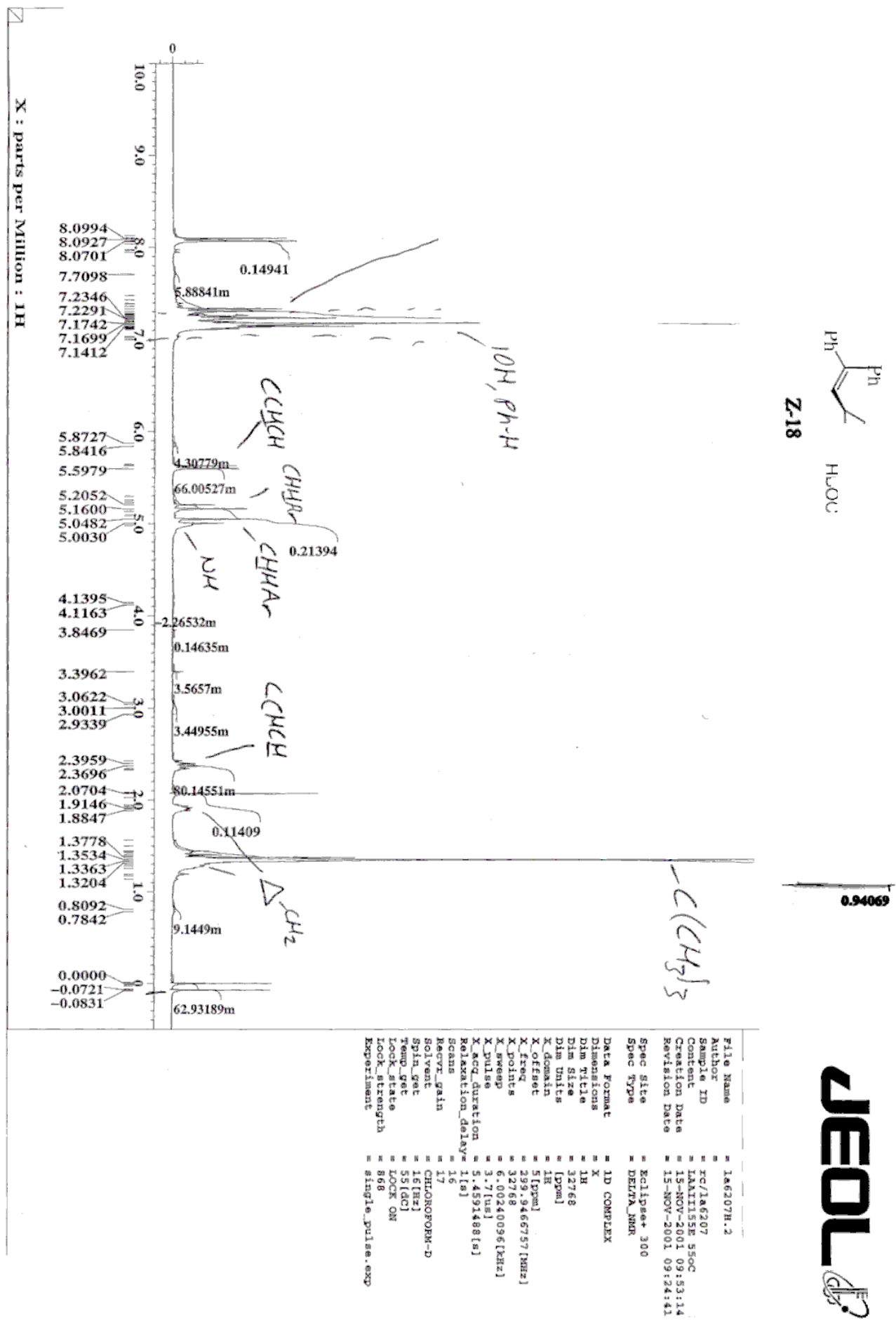




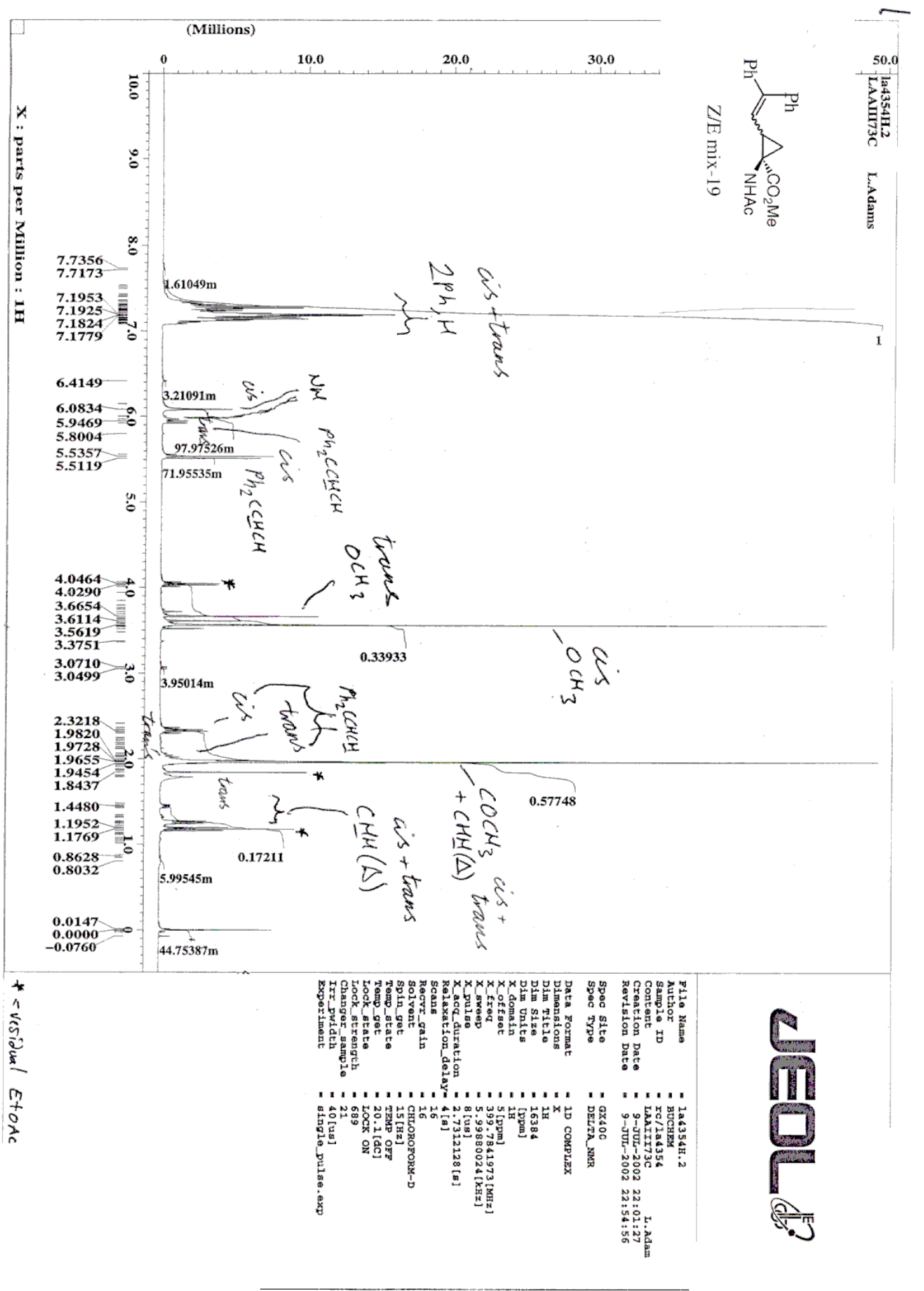




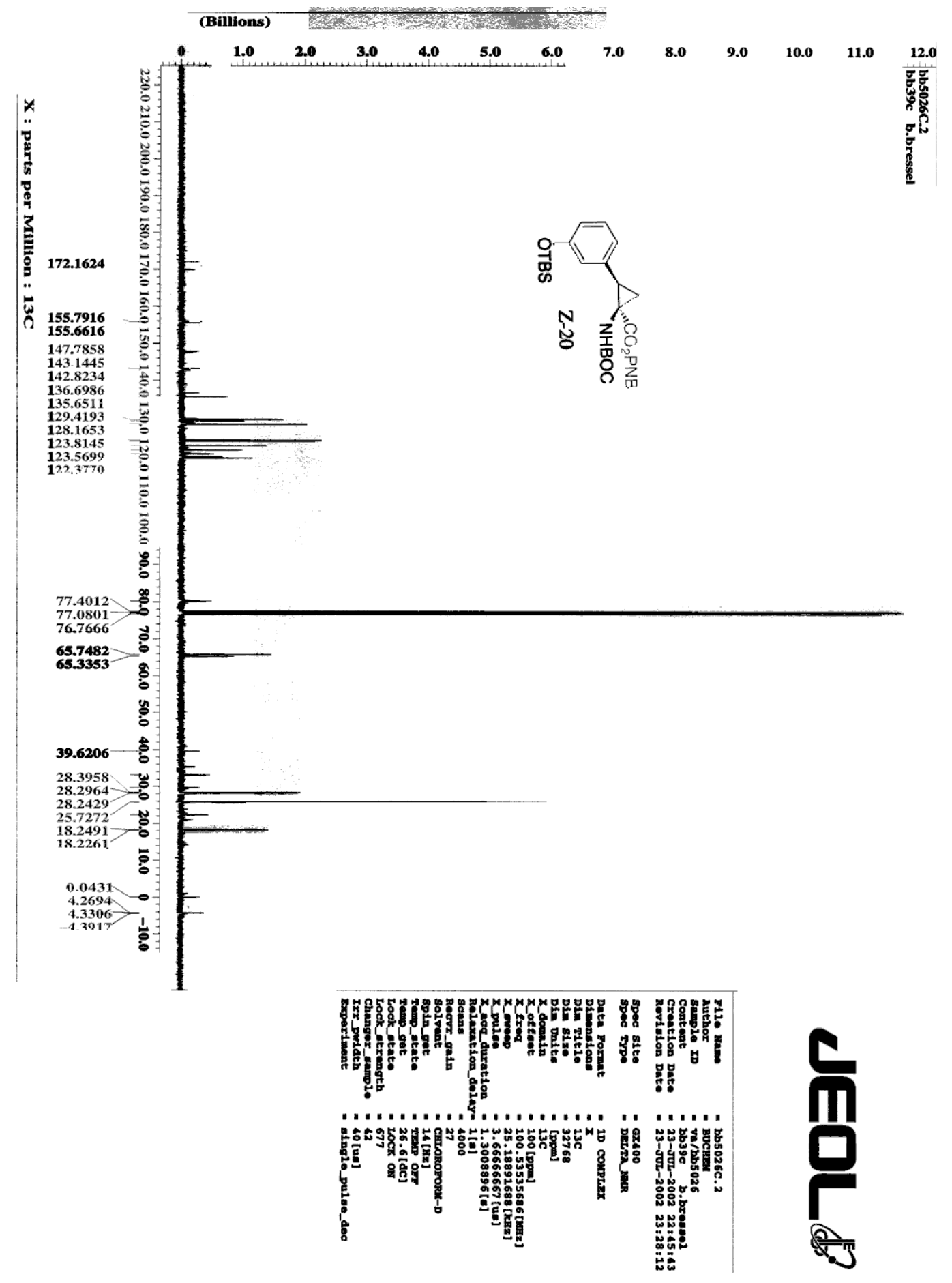




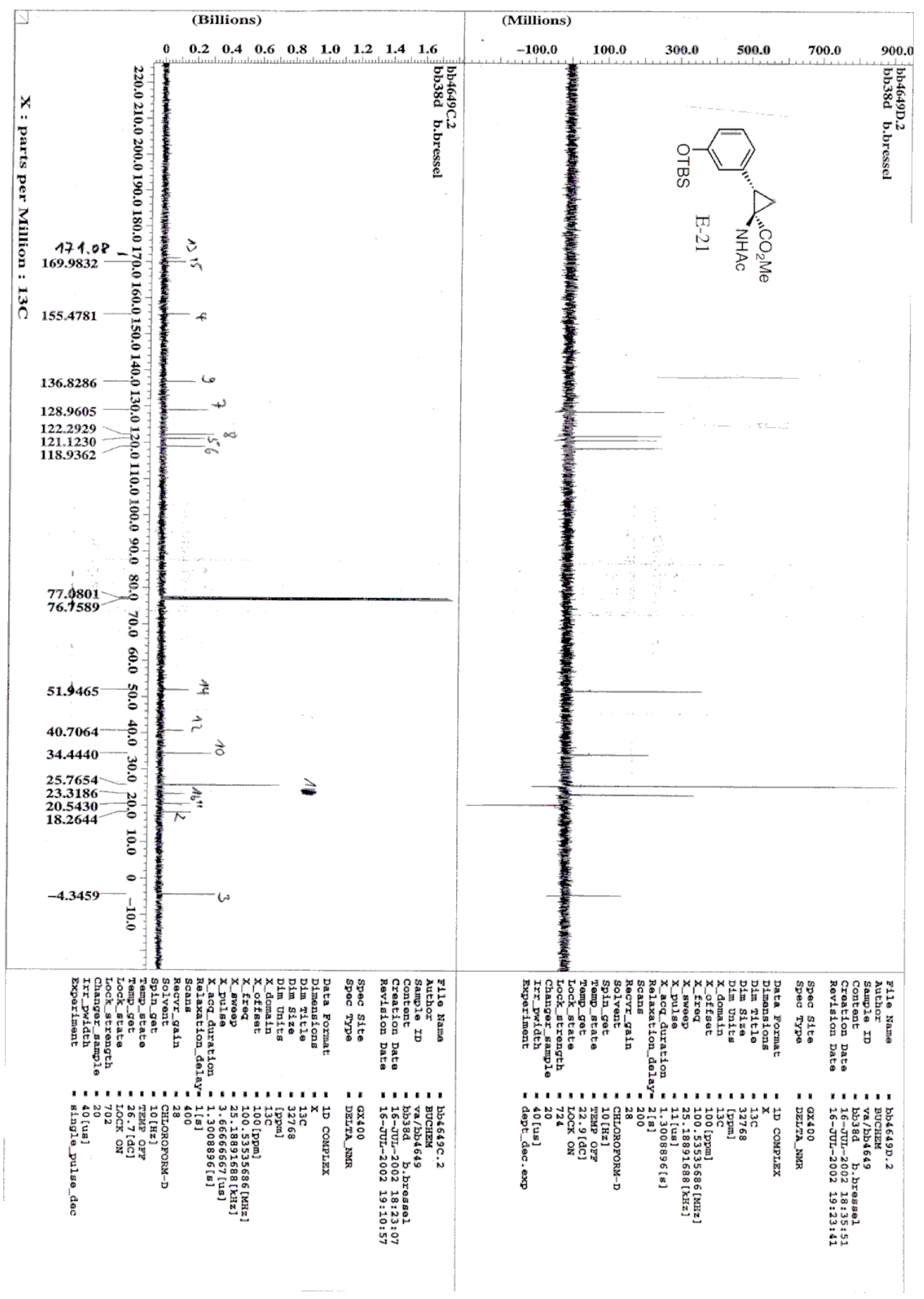




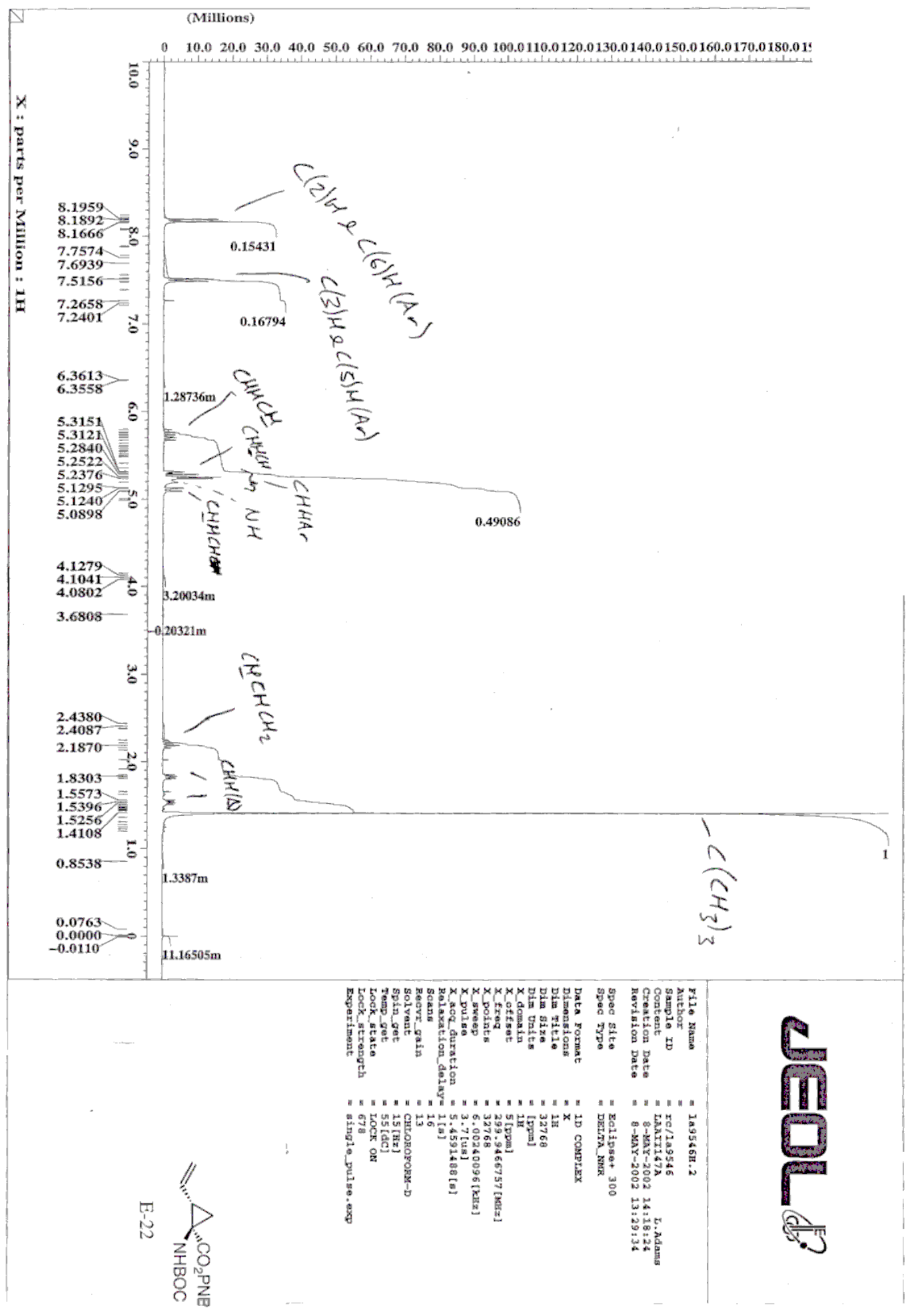




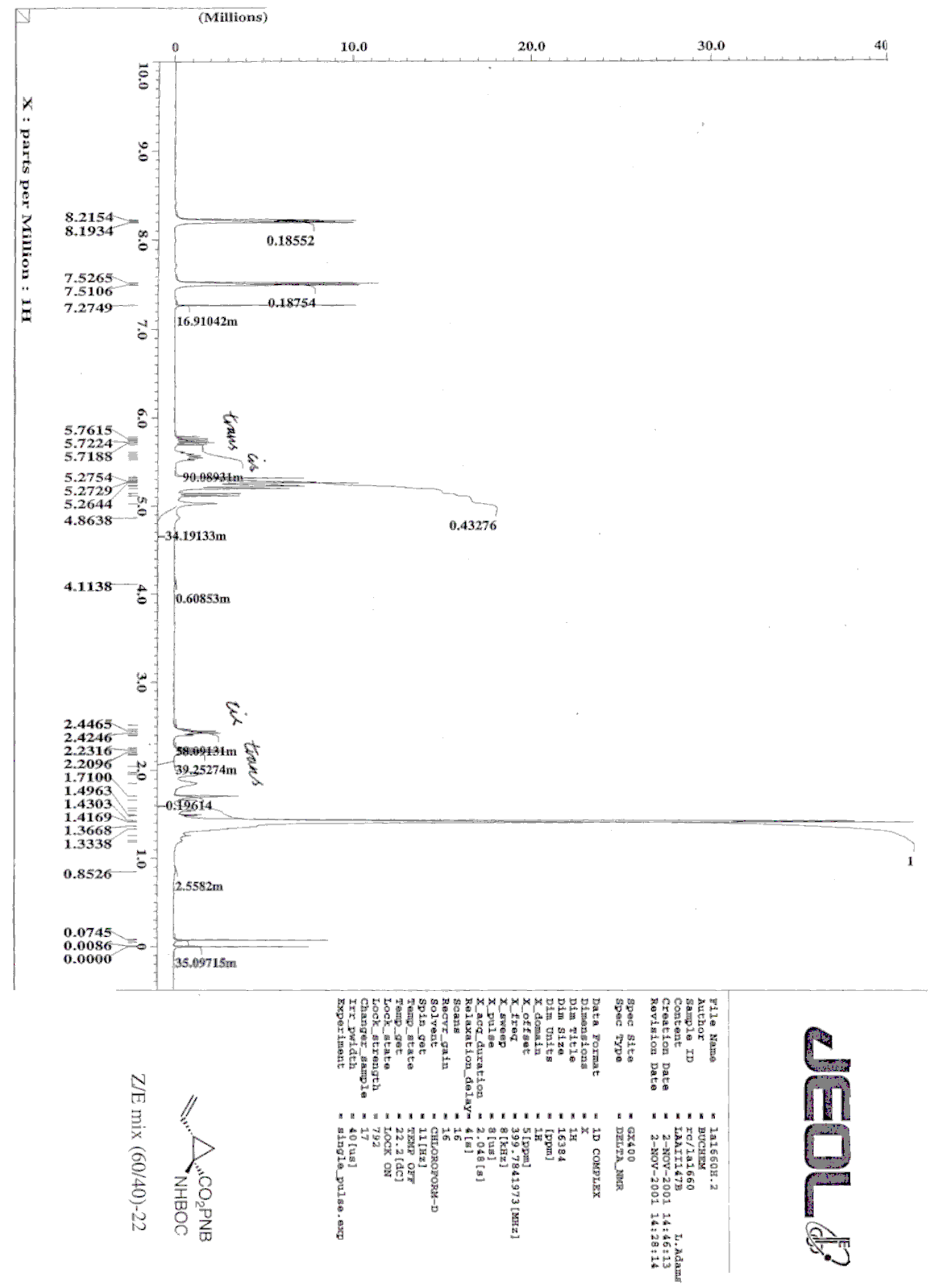




\section{References.}

(1) The solvent column is composed of activated alumina (A-2). For toluene a supported copper redox catalyst (Q-5 reactant) is also employed. See: Pangborn, A. B.; Giardello, M. A.; Grubbs, R. H.; Rosen, R. K.; Timmers, F. J. Organometallics 1996, 15, 1518.

(2) Zhu, Y.-F.; Yamazaki, T.; Tsang, J. W.; Lok, S.; Goodman, M. J. Org. Chem. 1992, 57, 1 074-1081

(3) Srivastava, V. P.; Roberts, M.; Holmes, T.; Stammer, C. H. J. Org. Chem. 1989, 54, 58665870.

(4) Ferreira, P. M. T.; Maia, H. L. S.; Monteiro, L. S. Tetrahedron Lett. 1999, 40, 4099-4102.

(5) McMahon, R. J.; Abelt, C. J.; Chapman, O. L.; Johnson, J. W.; Kreil, C. L.; LeRoux, J. P.; Mooring, A. M.; West, P. R. J. Am. Chem. Soc. 1987, 109, 2456-2469.

(6) Kabalka, G. W.; Maddox, J. T.; Boyas, E. J. Org. Chem. 1994, 59, 5530.

(7) Aggarwal, V. K.; Alonso, E.; Hynd, G.; Lydon, K. M.; Palmer, M. J.; Porcelloni, M.; Studley, J. R. Angew. Chem., Int. Ed. Engl. 2001, 40, 1430-1433.

(8) Ding, W. F.-X.; Xie, J. R.-Y.; Ji, G.-Z.; Jiang, X.-K. J. Chem. Res. (M) 1998, 1491.

(9) Grandi, R.; Marchesini, A.; Pagnoni, U. M.; Trave, R. J. Org. Chem. 1976, 41, 1755-1758.

(10) Nakatani, K.; Okamoto, A.; Saito, I. Tetrahedron Lett. 1996, 52, 9427-9446.

(11) The aldehyde was used in the following step without further purification.

(12) Aggarwal, V. K.; Alonso, E.; Bae, I.; Hynd, G.; Lydon, K. M.; Palmer, M. J.; Patel, M.; Porcelloni, M.; Richardson, J.; Stenson, R. A.; Studley, J. R.; Vasse, J.-L.; Winn, C. L. J. Am. Chem. Soc. 2003, 125, 10926-10940.

(13) Creary, X. Org. Synth. 1986, 64, 207-216.

(14) Srivastava, V. P.; Roberts, M.; Holmes, T.; Stammer, C. H. J. Org. Chem. 1989, 54, 58665870 .

(15) Ferreira, P. M. T.; Maia, H. L. S.; Monteiro, L. S. Tetrahedron Lett. 1999, 40, 4099-4102.

(16) Lenoy, C.; Dupas, G.; Bourguignon, J.; Queguiner, G. Tetrahedron 1994, 50, 1313513144.

(17) Davies, H. M. L.; Bruzinski, P.; Hutcheson, D. K.; Kong, N.; Fall, M. J. J. Am. Chem. Soc. 1996, 118, 6897-6907.

(18) Arenal, I.; Bernabé, M.; Fernández, A. E.; Izquierdo, M. L.; Penadés, S. J. Heterocycl. Chem. 1983, 20, 607-613.

(19) SMART diffractometer control software, Bruker Analytical X-ray Instruments Inc., Madison, WI, 1998. 
(20) SAINT integration software, Siemens Analytical X-ray Instruments Inc., Madison, WI, 1994.

(21) G. M. Sheldrick. SADABS: A program for absorption correction with the Siemens SMART system; University of Göttingen: Germany, 1996.

(22) SHELXTL program system version 5.1; Bruker Analytical X-ray Instruments Inc., Madison, WI, 1998.

(23) International Tables for Crystallography, Kluwer, Dordrecht, 1992, vol. C. 